The Astrophysical Journal SuPPlement Series, 85:119-136, 1993 March

(c) 1993. The American Astronomical Society. All rights reserved. Printed in U.S.A.

\title{
A SEARCH FOR OB ASSOCIATIONS NEAR SOUTHERN LONG-PERIOD CEPHEIDS. IV. XY CARINAE, GT CARINAE, WZ SAGITTARII, AND SW VELORUM
}

\author{
DAVID G. TURNER \\ Department of Astronomy, Saint Mary's University, Halifax, N.S., Canada, B3H 3C3; and Dominion Astrophysical Observatory \\ SIDNEY VAN DEN BERGH ${ }^{1}$ AND P. FRANK YOUNGER ${ }^{2}$ \\ Dominion Astrophysical Observatory, Herzberg Institute of Astrophysics, National Research Council of Canada, \\ 5071 West Saanich Road, Victoria, B.C., Canada, V8X 4M6
}

T. A. DANKS

Canadian Forces Meteorological and Oceanographic Centre, Maritime Command Headquarters, Fleet Mail Office, Halifax, N.S., Canada, B3K 2X0

AND

DOUGLAS FORBES

Department of Physics, Sir Wilfred Grenfell College, Memorial University, Corner Brook, Newfoundland, Canada, A2H 6P9

Received 1992 March 27; accepted 1992 June 5

\begin{abstract}
We report $U B V$ photometry for fields surrounding four long-period Cepheids in the Southern Milky Way. No potential early-type companions are detected around XY Car, which appears to be relatively isolated. However, an anonymous group of nine B-type and two possible FG supergiant stars are detected as possible companions to GT Car, despite its location at a seemingly improbably large distance of $10 \mathrm{kpc}$. WZ Sgr is found to lie in a sparse, previously undetected cluster, $1.8 \mathrm{kpc}$ distant, containing upward of 76 potential early-type member stars. Preliminary spectroscopic observations strengthen the case for cluster membership in this instance. SW Vel appears to belong to an older subgroup of the $2.5 \mathrm{kpc}$ distant Vel OB5 association described by Turner (1979), with $28 \mathrm{~B}$ star candidates in its immediate vicinity. Derived space reddenings and luminosities for WZ Sgr and SW Vel are consistent with their group membership, and the parameters of their associated stars, as well as those for the potential companions of GT Car, indicate that they are of comparable age to their associated Cepheid. Information is provided for follow-up studies of these stellar groups.
\end{abstract}

Subject headings: Cepheids - open clusters and associations: general

\section{INTRODUCTION}

This is the fourth in a series of papers describing a search for OB associations near southern hemisphere Cepheid variables with periods in excess of 11 days. Previous papers, with their corresponding Cepheid fields, are Paper I (van den Bergh, Brosterhus, \& Alcaino 1982) WZ Car, YZ Car, KK Cen, and OO Cen; Paper II (van den Bergh et al. 1983) CT Car, UU Mus, VZ Pup, SV Vel, and EZ Vel; and Paper III (van den Bergh, Younger, \& Turner 1985) U Car, XZ Car, QY Cen, VX Cru, and AA Nor. Here we describe the photoelectric and photographic photometry for stars lying in the fields of the Cepheids XY Car, GT Car, WZ Sgr, and SW Vel.

\section{OBSERVATIONS}

Table 1 gives $U B V$ data for skeleton photoelectric standard sequences observed in three of the Cepheid fields. Table 2 presents $U B V$ data for the more extensive photoelectric sequence surrounding WZ Sgr. Papers I and II contain details of the observing procedures used by van den Bergh to obtain the

\footnotetext{
${ }^{1}$ Visiting Astronomer, Cerro Tololo Inter-American Observatory, National Optical Astronomy Observatories, which is operated by the Association of Universities for Research in Astronomy, Inc. (AURA) under cooperative agreement with the National Science Foundation.

${ }^{2}$ Visiting Astronomer, Las Campanas Observatory.
}

skeleton sequences tabulated here. For the WZ Sgr field the data are compiled from the listed sources, all transformed to a common system closely tied to the Johnson system. This compilation involved corrections for small systematic errors detected in the faint star photometry of Turner (1984). The 7th mag O7 II(f) star HD 167659 (Walborn 1973) is included for the sake of completeness, although its brightness precluded its use in the photographic calibrations. The resulting photoelectric sequence is quite extensive compared with most other fields examined in this series. This was particularly important for the calibration of the iris measures, and the resulting external errors in the photographic photometry for this field are smaller than has been the case previously, despite the availability of only one plate in each color.

Brosterhus and Younger obtained photographic plates in $U$, $B$, and $V$ of fields centered on XY Car, GT Car, WZ Sgr, and SW Vel using the Las Campanas Observatory $0.9 \mathrm{~m}$ Swope Reflector, as summarized in Table 3. The plates for the XY Car and SW Vel fields were measured by Younger using the University of Victoria iris photometer, while the plates for the GT Car and WZ Sgr fields were measured by Danks and Turner using the partially automated iris photometer at Saint Mary's University (Reed, Turner, \& Scrimger 1986). The internal scatter of repeated iris measures for the same plate is typically no larger than $\pm 0.02 \mathrm{mag}$, while the color transforma- 
TABLE 1

PhotoeleCtric SeQuenCES fOR THE FieldS OF XY CARINAE, GT CARINAE, AND SW VELORUM

\begin{tabular}{|c|c|c|c|}
\hline Star & $V$ & $B-V$ & $U-B$ \\
\hline \multicolumn{4}{|c|}{ XY Car Field ${ }^{\mathrm{a}}$} \\
\hline A . & 10.10 & 0.80 & 0.38 \\
\hline B $\ldots \ldots$ & 12.12 & 1.49 & 1.52 \\
\hline $\mathrm{C}$ & 15.51 & 1.41 & \\
\hline D ....... & 14.47 & 0.83 & 0.26 \\
\hline E $\ldots \ldots$ & 14.83 & 0.43 & 0.26 \\
\hline$F \ldots \ldots$ & 13.06 & 0.76 & 0.35 \\
\hline$G \ldots \ldots$ & 13.27 & 0.45 & 0.44 \\
\hline $\mathrm{H} \ldots \ldots$ & 13.00 & 1.71 & 1.95 \\
\hline \multicolumn{4}{|c|}{ GT Car Field ${ }^{\mathrm{b}}$} \\
\hline A $\ldots .$. & 8.29 & 0.02 & -0.03 \\
\hline B $\ldots \ldots$ & 10.92 & 0.20 & -0.78 \\
\hline $\mathrm{C} \ldots \ldots$ & 12.47 & 0.32 & -0.04 \\
\hline $\mathrm{D} \ldots \ldots$ & 12.84 & 0.35 & 0.13 \\
\hline$E \ldots \ldots$ & 11.65 & 1.44 & 1.56 \\
\hline$F \ldots \ldots$ & 13.09 & 0.65 & 0.21 \\
\hline${ }^{*} \mathrm{G} \ldots \ldots$ & 13.18 & 1.27 & 0.98 \\
\hline $\mathrm{H} \ldots \ldots$ & 13.63 & 0.33 & 0.22 \\
\hline I $\ldots . .$. & 15.17 & 1.27 & $\ldots$ \\
\hline \multicolumn{4}{|c|}{ SW Vel Field ${ }^{c}$} \\
\hline A $\ldots .$. & 10.52 & $0.11^{+}$ & 0.04 \\
\hline B $\ldots \ldots$ & 11.89 & 0.54 & 0.10 \\
\hline$C \ldots \ldots$ & 12.78 & 0.33 & 0.23 \\
\hline D ....... & 12.85 & 0.52 & 0.05 \\
\hline $\mathrm{E} \ldots \ldots$ & 13.31 & 0.43 & 0.33 \\
\hline$F \ldots \ldots$ & 10.56 & 1.76 & 2.07 \\
\hline$G \ldots \ldots$ & 11.41 & 1.12 & 0.74 \\
\hline $\mathrm{H} \ldots \ldots$ & 14.66 & 1.11 & 0.73 \\
\hline I $\ldots \ldots$ & 14.71 & 1.16 & $\ldots$ \\
\hline
\end{tabular}

a Observations by van den Bergh UT 1979 March 23 with CTIO $1.5 \mathrm{~m}$ telescope.

${ }^{b}$ Observations by van den Bergh UT 1978 April 8/9 with CTIO $1.5 \mathrm{~m}$ telescope.

c Observations by van den Bergh UT 1979 March 22 with CTIO $1.5 \mathrm{~m}$ telescope.

tions are similar to those quoted in Paper I. Typical external errors in the iris photometry are also not very different from those quoted in earlier papers in this series.

According to Weaver (1962), the mean external errors for single iris measures of star images lying well above the plate limits on good quality, large reflector photographs should be of order \pm 0.03 to $\pm 0.04 \mathrm{mag}$. Turner \& Welch (1989) have confirmed this for iris photometry at Saint Mary's University and find typical errors of measurement no larger than about \pm 0.03 mag in $U$ and $B$, and \pm 0.04 mag in $V$, for single, uncrowded images of stars several magnitudes brighter than the plate limits. The photographic photometry for stars in the WZ Sgr field is of comparable accuracy; a formal comparison of the photographic and photoelectric magnitudes and colors for all uncrowded standards yields external errors of \pm 0.04 mag in $V$ and $\pm 0.03 \mathrm{mag}$ in $(B-V)$ and $(U-B)$. The color-color
TABLE 2

Photoelectric SEQUENCE FOR THE FIELd OF WZ SAgitTARII

\begin{tabular}{|c|c|c|c|c|c|}
\hline Star & V & $B-V$ & $U-B$ & $n$ & References \\
\hline HD 167659 & 7.39 & 0.21 & -0.74 & & $1,2,3$ \\
\hline$\ldots$. & 9.40 & 0.36 & -0.58 & 3 & 1,2 \\
\hline$\ldots \ldots \ldots$ & 9.57 & 0.54 & -0.35 & 3 & $1,2,4$ \\
\hline C. & 9.67 & 1.10 & 0.86 & 1 & 4 \\
\hline${ }^{*} \mathrm{D}$ & 9.82 & 0.27 & -0.39 & 3 & 1,2 \\
\hline$*^{*} \mathrm{E}$ & 10.49 & 0.25 & -0.43 & 2 & 5 \\
\hline $\mathrm{F}$ & 10.66 & 0.77 & 0.49 & 1 & 4 \\
\hline G & 10.85 & 0.47 & -0.37 & 1 & 4 \\
\hline $\mathrm{H}$ & 11.31 & 0.73 & -0.20 & 1 & 4 \\
\hline I... & 11.73 & 0.70 & 0.13 & 6 & 4,6 \\
\hline $\mathrm{J} .$. & 12.04 & 1.39 & 1.20 & 2 & 5 \\
\hline${ }^{*} \mathrm{~K}$ & 12.17 & 0.39 & -0.03 & 6 & 4,6 \\
\hline L ... & 12.27 & 0.77 & 0.29 & 2 & 5 \\
\hline ........ & 12.42 & 0.33 & 0.14 & 2 & 5 \\
\hline $\mathrm{N}$ & 12.63 & 1.71 & 1.82: & 2 & 5 \\
\hline${ }^{*} \mathrm{O}$ & 12.76 & 0.41 & -0.04 & 9 & 5,6 \\
\hline${ }^{*} \mathrm{P}$ & 12.97 & 0.31 & -0.09 & 8 & 4,6 \\
\hline${ }^{*} \mathrm{Q}$ & 13.35: & $0.44:$ & 0.15 : & 8 & 4,6 \\
\hline $\mathrm{R}$ & 13.35 & 0.63 & 0.55 & 1 & 5 \\
\hline $\mathrm{S}$ & 13.42 & 0.61 & 0.51 & 2 & 5 \\
\hline$\ldots \ldots \ldots$ & 13.60 & 0.52 & 0.21 & 10 & 5,6 \\
\hline $\mathrm{U}$ & 13.67 & 1.67 & 1.64 & 4 & 6 \\
\hline${ }^{*} \mathrm{~V}$ & 13.75 & 0.56 & 0.15 & 9 & $4,5,6$ \\
\hline$* \mathrm{~W}$ & 14.06 & 0.52 & 0.34 & 9 & 5,6 \\
\hline${ }^{*} \mathrm{X}$ & 14.26 & 0.70 & 0.26 & 1 & 6 \\
\hline $\mathrm{Y}$ & 14.29 & 1.56: & 1.40: & 2 & 6 \\
\hline $\mathrm{Z}$ & 15.05 & 0.59 : & $0.24:$ & 5 & 6 \\
\hline
\end{tabular}

REFERENCES.-(1) Klare \& Neckel 1977; (2) Schild, Garrison, \& Hiltner 1983; (3) Hiltner 1956; (4) Observations by van den Bergh UT 1978 April 12/13 with CTIO $1.5 \mathrm{~m}$ telescope; (5) Observations by Forbes 1984 April with LCO $0.6 \mathrm{~m}$ telescope; (6) Observations by Turner 1977 August and 1981 April/May with LCO $0.6 \mathrm{~m}$ telescope.

TABLE 3

Photographic Plate Data

\begin{tabular}{|c|c|c|c|c|c|}
\hline Star & UT Date & Emulsion & Filter & $\begin{array}{c}\text { Exposure } \\
\text { Time } \\
\text { (minutes) }\end{array}$ & $\begin{array}{c}\text { Limiting } \\
\text { Measured } \\
\text { Magnitude }\end{array}$ \\
\hline XY Car .... & $\begin{array}{l}1982.02 .20 \\
1982.02 .20 \\
1982.02 .20\end{array}$ & $\begin{array}{l}103 a-D \\
103 a-O \\
103 a-O\end{array}$ & $\begin{array}{l}\text { GG495 } \\
\text { GG385 } \\
\text { UG1 }\end{array}$ & $\begin{array}{l}30 \\
30 \\
90\end{array}$ & $\begin{array}{l}15.3 \\
17.5 \\
17.2\end{array}$ \\
\hline GT Car & $\begin{array}{l}1982.02 .25 \\
1979.03 .31 \\
1978.05 .12 \\
1982.02 .26\end{array}$ & $\begin{array}{l}103 a-D \\
103 a-O \\
103 a-O \\
103 a-O\end{array}$ & $\begin{array}{l}\text { GG495 } \\
\text { GG385 } \\
\text { UG1 } \\
\text { UG1 }\end{array}$ & $\begin{array}{l}30 \\
30 \\
68 \\
90\end{array}$ & $\begin{array}{l}14.6 \\
15.2 \\
15.3 \\
15.3\end{array}$ \\
\hline WZ Sgr. & $\begin{array}{l}1978.05 .09 \\
1978.05 .09 \\
1978.05 .09\end{array}$ & $\begin{array}{l}103 a-D \\
103 a-O \\
103 a-O\end{array}$ & $\begin{array}{l}\text { GG495 } \\
\text { GG385 } \\
\text { UG1 }\end{array}$ & $\begin{array}{l}30 \\
30 \\
90\end{array}$ & $\begin{array}{l}16.0 \\
16.9 \\
17.9\end{array}$ \\
\hline SM Vel & $\begin{array}{l}1982.02 .21 \\
1982.02 .21 \\
1982.02 .28\end{array}$ & $\begin{array}{l}103 a-D \\
103 a-O \\
103 a-O\end{array}$ & $\begin{array}{l}\text { GG495 } \\
\text { GG385 } \\
\text { UG1 }\end{array}$ & $\begin{array}{l}30 \\
30 \\
90\end{array}$ & $\begin{array}{l}15.2 \\
16.4 \\
17.0\end{array}$ \\
\hline
\end{tabular}

NOTES._-1978 and 1979 observations by Brosterhus; 1982 observations by Younger. 


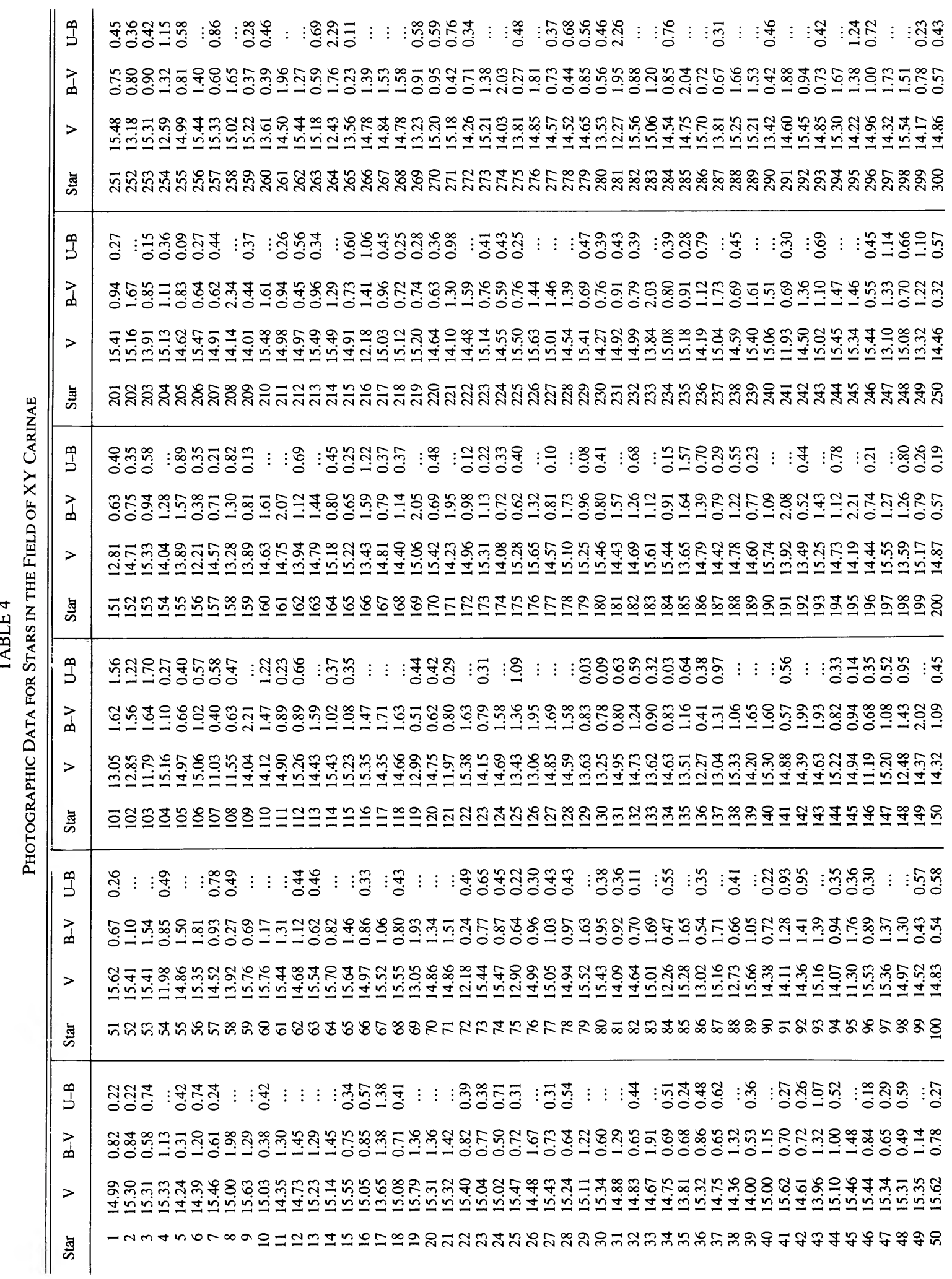




\begin{tabular}{|c|c|}
\hline jి & 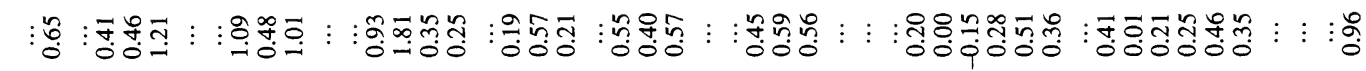 \\
\hline 1 & 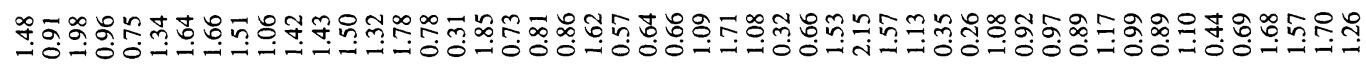 \\
\hline & 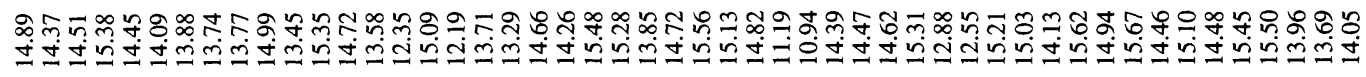 \\
\hline 焉 & $\begin{array}{l}b \infty \\
0 \infty \infty \\
0 \infty\end{array}$ \\
\hline If & 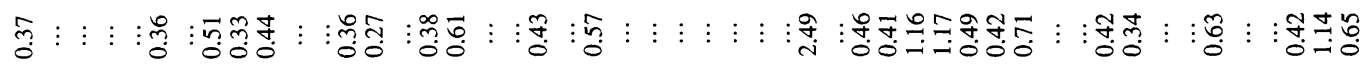 \\
\hline के & 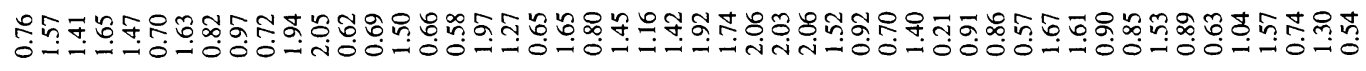 \\
\hline$>$ & 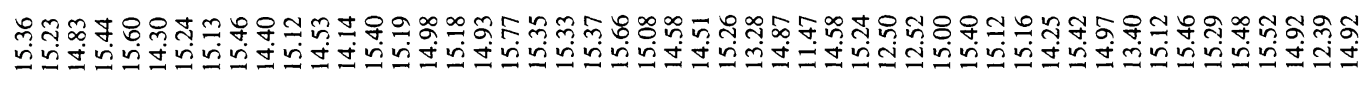 \\
\hline 急 & దิ \\
\hline 党 & : \\
\hline m & 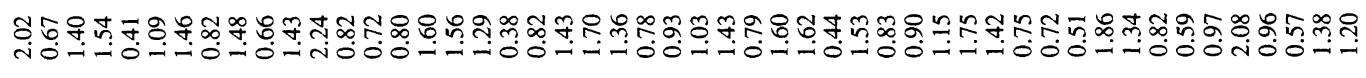 \\
\hline$>$ & 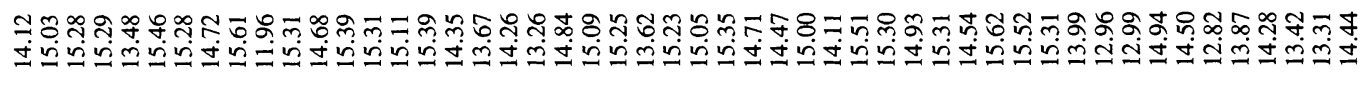 \\
\hline 氙 & 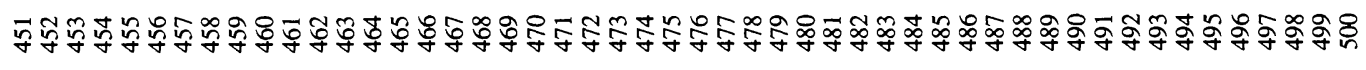 \\
\hline 乌ै & 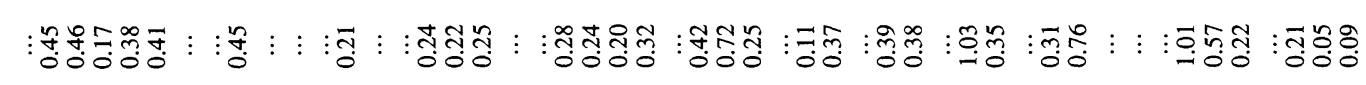 \\
\hline 宙 & 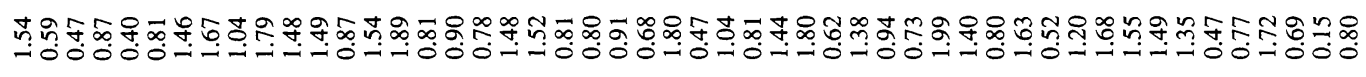 \\
\hline$>$ & 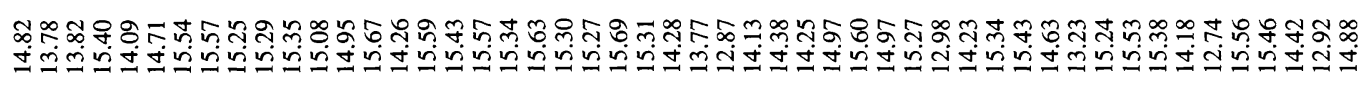 \\
\hline 壳 & 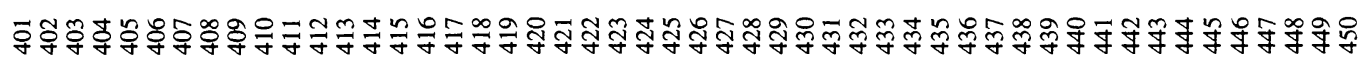 \\
\hline 乌े & 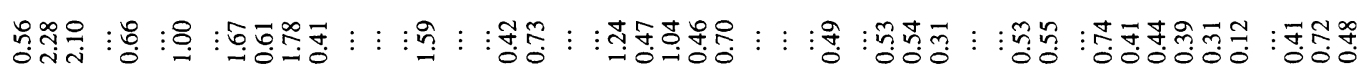 \\
\hline 殅 & \& \\
\hline$>$ & 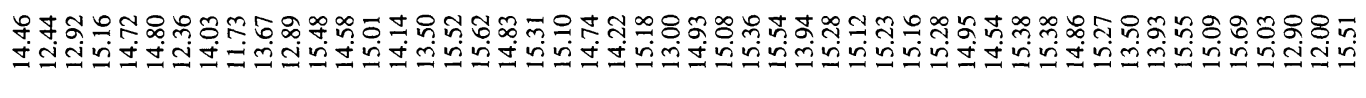 \\
\hline 惡 & 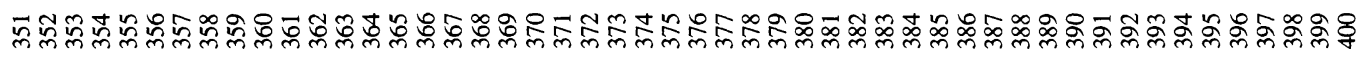 \\
\hline$\stackrel{p}{p}$ & 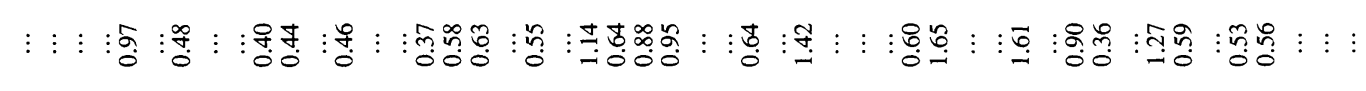 \\
\hline मे & 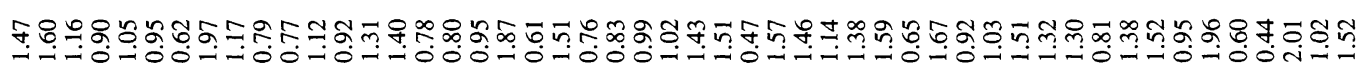 \\
\hline$>$ & 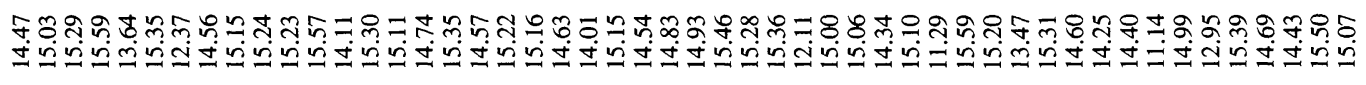 \\
\hline s. & 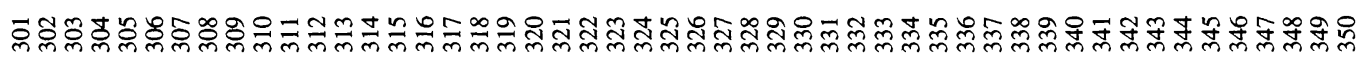 \\
\hline
\end{tabular}


TABLE 4-Continued

\begin{tabular}{cccc|cccc|cccc}
\hline \hline Star & V & B-V & U-B & Star & V & B-V & U-B & Star & V & B-V & U-B \\
\hline & & & & & & & & & & \\
601 & 14.75 & 0.72 & 0.23 & 606 & 15.62 & 1.49 & $\ldots$ & 611 & 13.42 & 0.14 & -0.10 \\
602 & 14.77 & 1.49 & $\ldots$ & 607 & 15.32 & 0.61 & 0.44 & 612 & 14.91 & 0.76 & 0.30 \\
603 & 14.87 & 1.00 & 0.39 & 608 & 15.61 & 1.02 & 0.19 & 613 & 14.74 & 0.40 & 0.38 \\
604 & 14.99 & 1.51 & $\ldots$ & 609 & 15.07 & 1.31 & $\ldots$ & 614 & 15.66 & 0.69 & 0.42 \\
605 & 15.01 & 1.45 & $\ldots$ & 610 & 15.68 & 1.14 & $\ldots$ & 615 & 15.61 & 0.95 & 0.14 \\
\hline
\end{tabular}

diagram for this field (see Fig. 8) confirms these values, since color errors of precisely this amount can account for the scatter of data points below the reddening line drawn from the A2 star "kink" in the intrinsic color relation. The errors increase in size for faint stars near the survey limits, many of which lie within a few magnitudes of the plate limits and are also near the calibration limits.

The uncertainties in the data for the three other Cepheid fields appear to be slightly larger as well [e.g., $\pm 0.05, \pm 0.07$, and $\pm 0.07 \mathrm{mag}$ for $V,(B-V)$, and $(U-B)$, respectively, for stars in the GT Car field ], although such estimates also contain a sizable contribution from rather large uncertainties in the photometry of the standards (which represent single observations). The true external uncertainties in the photographic values of $V,(B-V)$, and $(U-B)$ for these three fields are probably no larger than about $\pm 0.05 \mathrm{mag}$, except for stars lying well outside the range of the magnitude and color calibrations.

It was possible to obtain spectra of two cluster candidate stars in the WZ Sgr field using the RCA CCD camera on the Cassegrain spectrograph of the $1.8 \mathrm{~m}$ telescope at the Dominion Astrophysical Observatory on two nights in 1991 August. The spectra have a wavelength coverage of $978 \AA$ centered on $4200 \AA$ at a dispersion of $60 \AA \mathrm{mm}^{-1}$, and were included in a more extensive program involving spectroscopy of cluster $B$ stars. Details on their reduction and measurement are given by Turner, Forbes, \& Pedreros (1992). Spectral classifications, radial velocities, and estimates of projected rotational velocities for these stars are given later in Table 7.

\section{DISCUSSION}

\subsection{XY Carinae}

$\mathrm{XY}$ Car is a 12.437 Cepheid with $\langle V\rangle=9.294$, and $\langle B\rangle-$ $\langle V\rangle=1.215$ (Caldwell \& Coulson 1987). The period-luminosity and period-color relations of van den Bergh (1977) predict values of $\left\langle M_{V}\right\rangle=-4.35 \pm 0.26$ and $(\langle B\rangle-\langle V\rangle)_{0}=0.80 \pm$ 0.06 for a Cepheid with this period, which result in estimates of $E_{B-V}(B 0)=0.45 \pm 0.06$ and $V-M_{V}=13.64 \pm 0.26$ for XY Car. The predicted distance modulus is 12.25 , corresponding to a distance of $2.8 \mathrm{kpc}$.

The Cepheid lies nearly $4^{\circ}$ below the Galactic plane in a rather sparse and uninteresting portion of Carina about $2^{\circ}$ east of the bright cluster IC 2602 . The closest bright star, $4^{\prime}$ southwest of XY Car (see Fig. 1), is HD 95752 (also LSS 2104 of Stephenson \& Sanduleak 1971), which is classified as A7 Ib-II by Houk \& Cowley (1975) and A9 Ib-II by Stephenson \& Sanduleak (1971). There is another A-type supergiant in the general field, namely LSS 2061, classified as A9 II by Stephen- son \& Sanduleak (1971). Although their predicted luminosities are comparable to that expected for XY Car, and A-type Ib and II supergiants are expected to be of similar evolutionary status to long-period Cepheids, both stars are 1-2 mag brighter than the Cepheid. A common distance for all three stars therefore appears unlikely.

Stellar images lying within a $28^{\prime}$ diameter field centered on $\mathrm{XY}$ Car were measured to determine $V, B-V$, and $U-B$ for 378 stars and $V$ and $B-V$ only for an additional 237 stars. The data are given in Table 4 , and program stars and photoelectric standards for the field are identified in Figure 1 (Plate 1-4). The color-magnitude diagram for all 623 objects is presented in Figure 2, where the main sequence, corrected for the estimated distance and reddening of XY Car, is shown as a solid line. Most of the blue stars in the diagram lie well to the right of the plotted main sequence.

The color-color diagram for stars near XY Car is shown in Figure 3, with the locus for unreddened zero-age mainsequence (ZAMS) stars plotted as a solid line. A few of the blue stars appear to be late B dwarfs which are of comparable reddening to XY Car, but their distances inferred from ZAMS fitting are significantly less than $2.8 \mathrm{kpc}$. Also, most of the stars

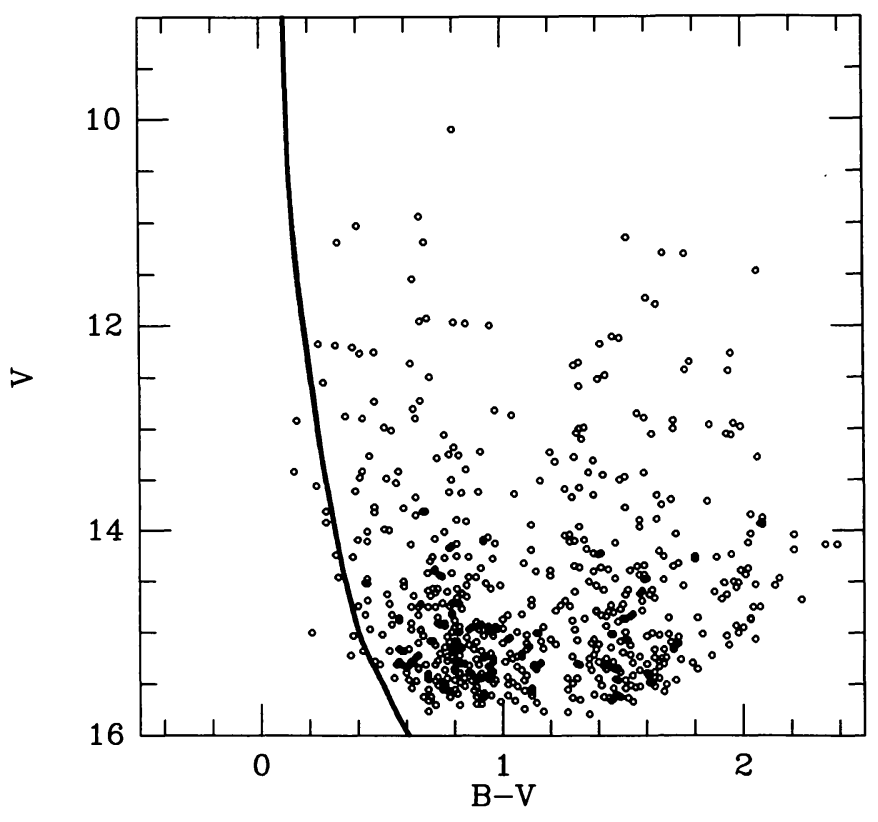

FIG. 2.-Color-magnitude diagram for stars in the XY Car field. The ZAMS is plotted for the expected parameters of the Cepheid. 


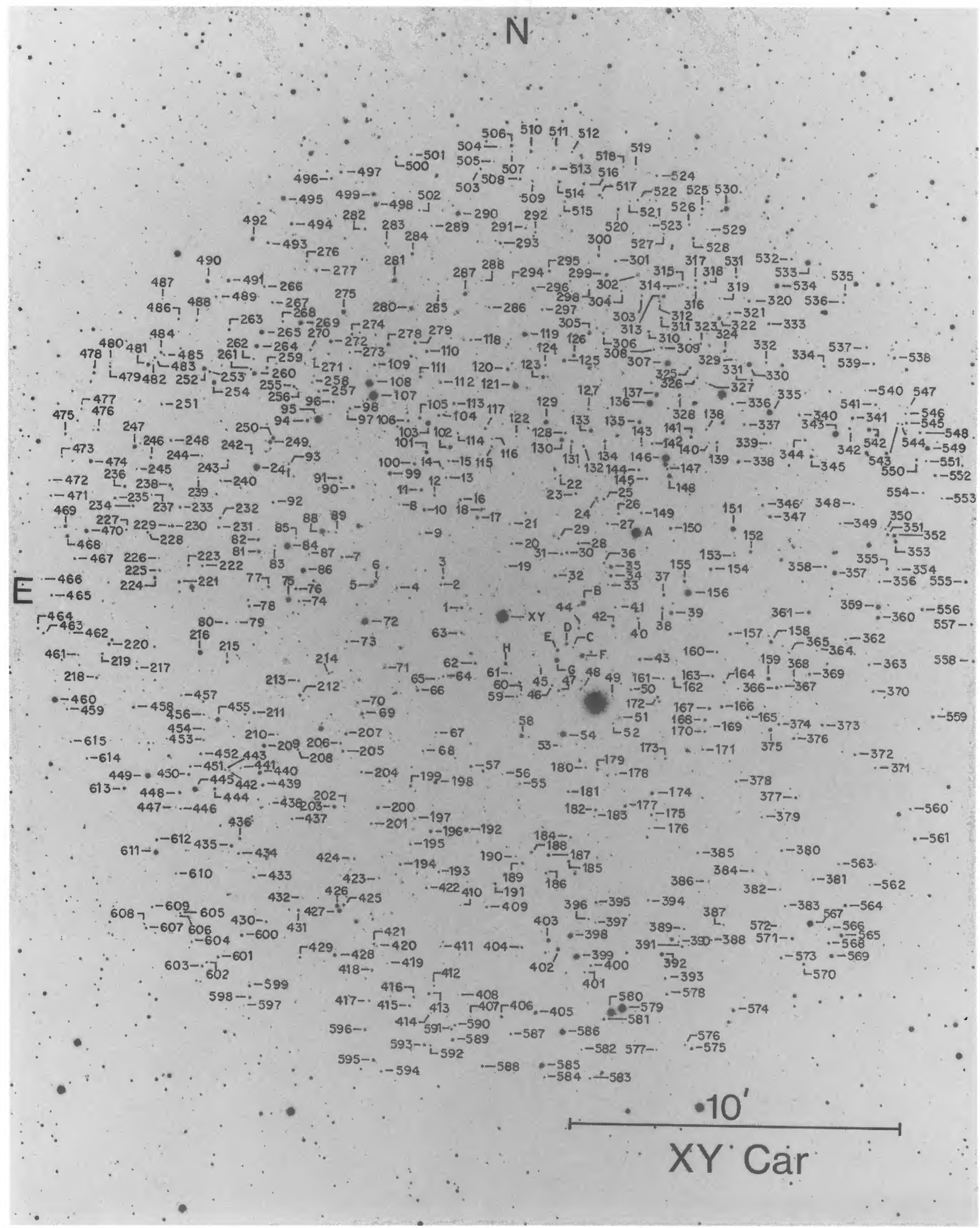

FIG. 1.-Identification chart in blue light for standard stars (letters) and photographically measured stars (numbers) in the field of XY Carinae (XY)

TURNER ET AL. ( see $85 ; 123$ ) 


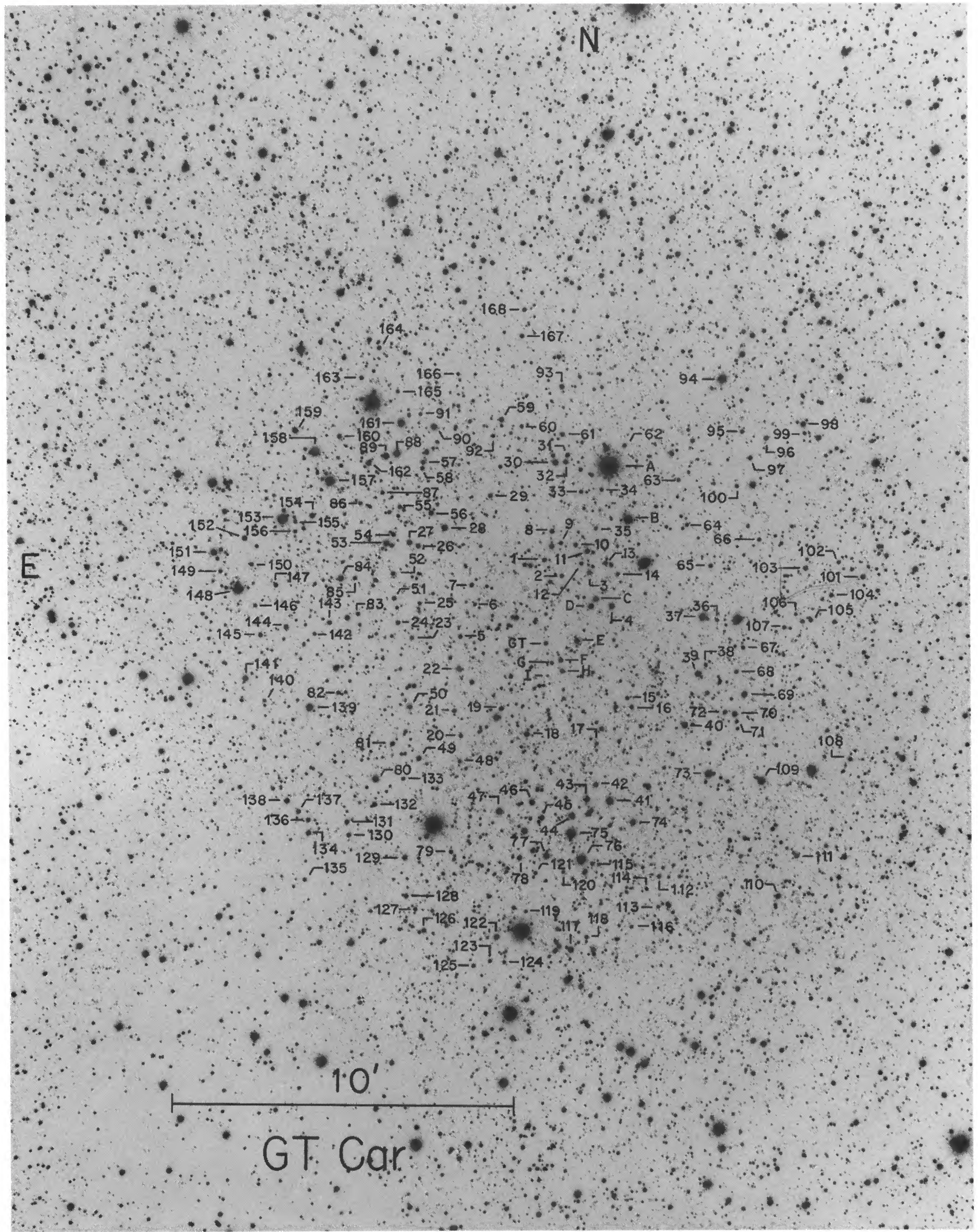

FIG. 4.-Identification chart in blue light for standard stars (letters) and photographically measured stars (numbers) in the field of GT Carinae (GT) 


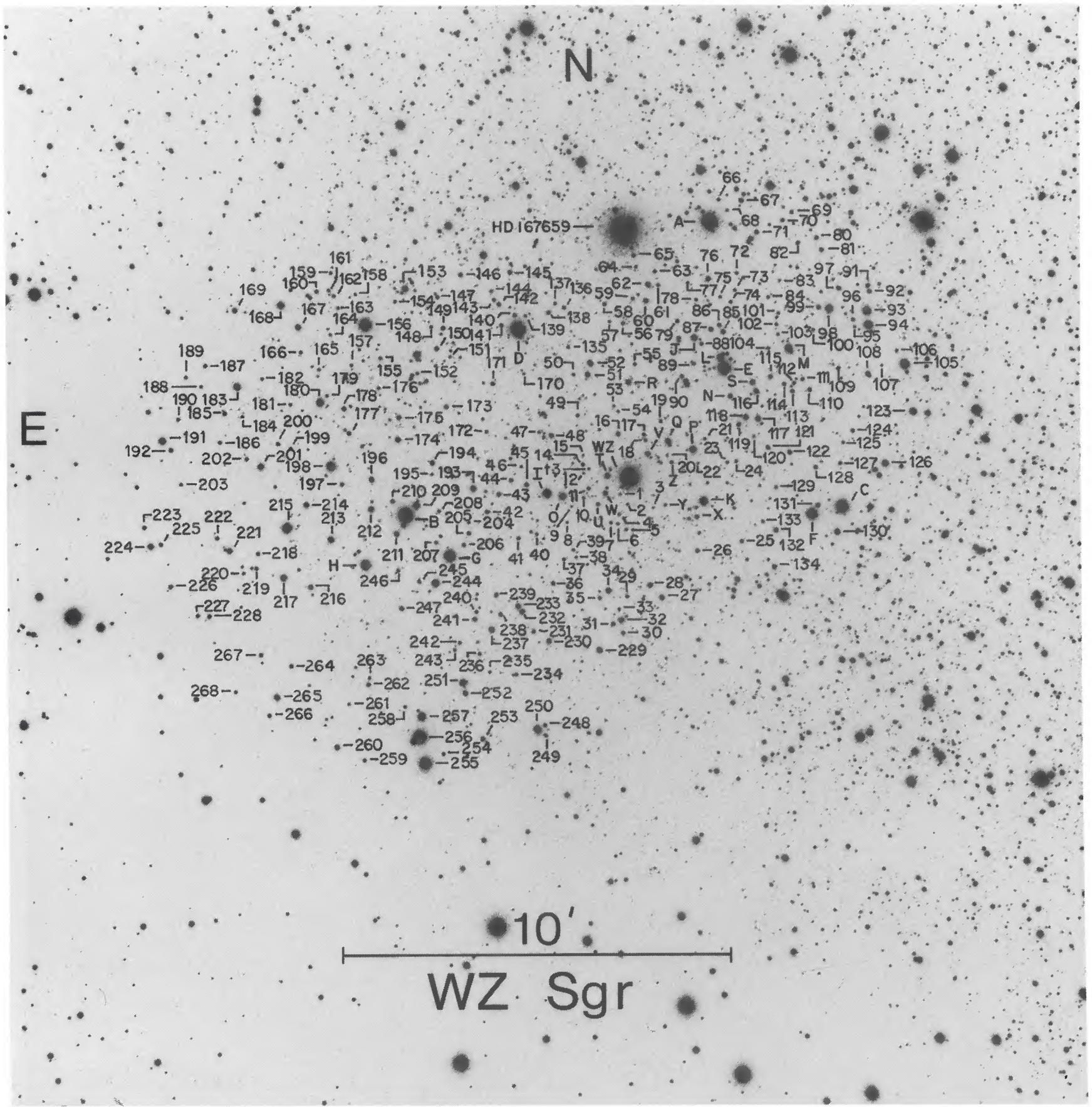

FIG. 7.-Identification chart in blue light for standard stars (letters) and photographically measured stars ( numbers) in the field of WZ Sagittarii (WZ) 
PLATE 4

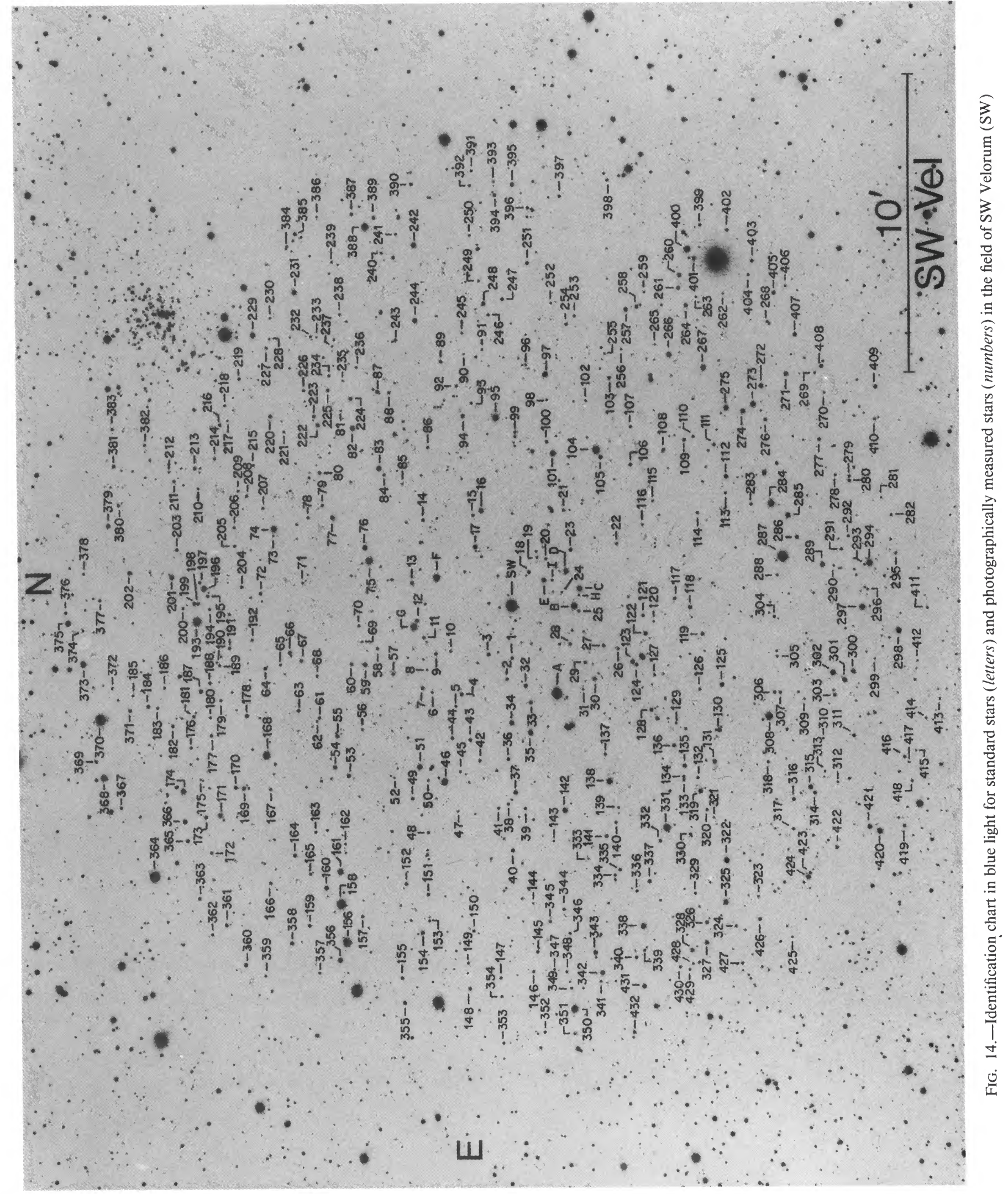




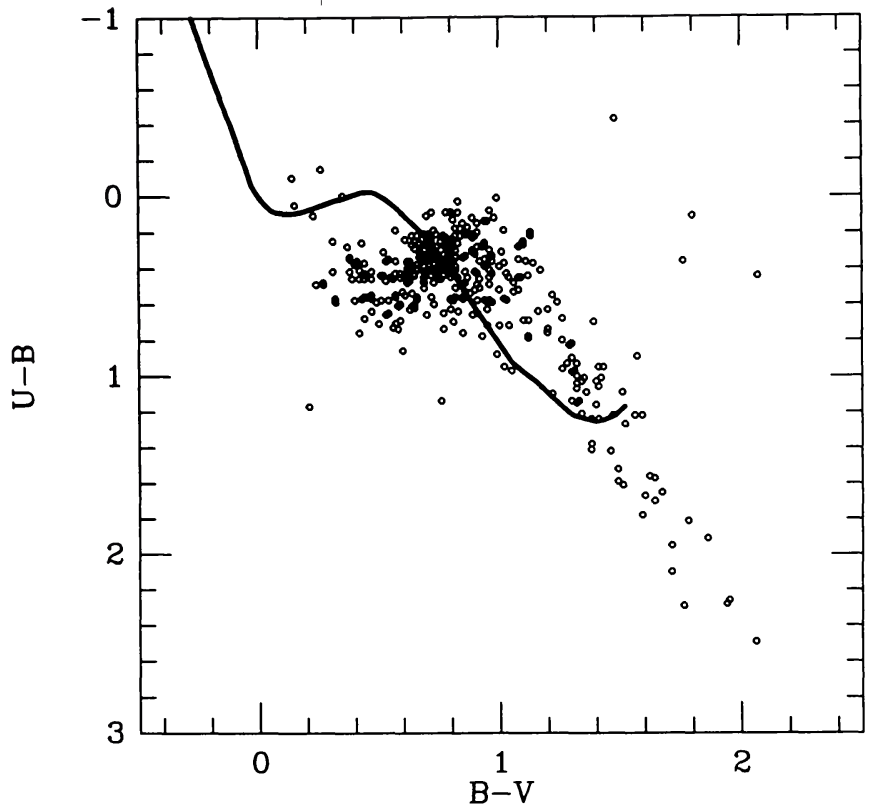

FIG. 3.-Color-color plot for stars in the XY Car field. The solid line is the intrinsic relation for dwarfs of solar metallicity.

in this field appear to be $\mathrm{A}$ and $\mathrm{F}$ stars with photometric reddenings smaller than $E_{B-V}=0.45$. No particular clustering of points outside the expected field star distribution is seen, and there is no evidence for the existence in this field of any middle to early B-type stars which might be associated with XY Car. The small number of late B star candidates detected here seem to be both less reddened and less distant than expected for objects associated with XY Car.

\subsection{GT Carinae}

GT Car is a $13^{\mathrm{d}} 162$ Cepheid with $\langle V\rangle=12.91,\langle B\rangle-$ $\langle V\rangle=1.44$, and $\langle U\rangle-\langle B\rangle=1.09$ (Madore 1975). It lies close to the Galactic plane in a region of relatively uniform extinction between the great Carina nebula (NGC 3372) and the rich open cluster NGC 3532 to its immediate northeast. The Cepheid itself lies $20^{\prime}$ northeast of U Car (Paper III) and $28^{\prime}$ southeast of the open cluster $\operatorname{Tr} 17$ (Sher 1964), both of which lie within the field of the photographic plates. The photoelectric sequences available for these fields were therefore used to supplement the skeleton sequence of Table 1 in calibrating the photographic measures. The plate exposures for this field are deep enough that many stars near the Cepheid could not be measured owing to crowding or to very red colors. Photographic data for the 168 uncrowded stars which could be measured in all three colors are presented in Table 5 . The stars are identified in Figure 4 (Plate 2).

The period-luminosity and period-color relations of van den Bergh (1977) predict values of $\left\langle M_{V}\right\rangle=-4.43 \pm 0.26$ and $(\langle B\rangle-\langle V\rangle)_{0}=0.81 \pm 0.06$ for $\mathrm{GT}$ Car based upon its period. Combined with the Madore data, these values imply $E_{B-V}(B 0)=0.69$ and $V-M_{V}=17.34$ for GT Car, corresponding to a distance modulus of 15.13 and a distance in excess of $10 \mathrm{kpc}$ ! The period of GT Car also implies an evolutionary age of about $3.5( \pm 0.7) \times 10^{7}$ yr (see Kippenhahn \& Smith 1969), which corresponds to a main-sequence turnoff near $(B-V)_{0}=-0.20$ (spectral type B3). Unfortunately, B3 stars reddened by $E_{B-V}=0.69$ lie close to the F star hump in the $U B V$ color-color diagram, where they would be difficult to separate from unreddened foreground stars. B3 stars on the ZAMS at the distance of GT Car would also be fainter than $V=16$, nearly 2 mag below the survey limit. Initial expectations for the detection of physical companions to GT Car were therefore decidedly pessimistic.

Figure 5 is a color-color diagram for the data in Tables 1 and 5. It is evident that a small range of reddening occurs within the GT Car field. The minimum reddening of $E_{B-V} \approx 0.05$ agrees with the small foreground reddening of $E_{B-V}=0.04 \pm 0.02$ found by Fernandez \& Salgado (1980) for the nearby cluster NGC 3532. Most stars identified photometrically as O- or Btype have reddenings of $E_{B-V} \approx 0.3$ to 0.5 , but there is a small group of nine B-type stars of larger reddening. Our attention was drawn to these stars by the fact that they are reddened to the same extent as that predicted for GT Car, they appear as a distinct clump at $V \approx 14$ in the color-magnitude diagram plotted in Figure 6, their inferred intrinsic colors are exactly those of B3 stars, and similar features are not seen in the fields of the spatially adjacent Cepheids U Car and XZ Car (Paper III), indicating that they are not simply foreground $\mathrm{F}$ stars with colors similar to those of reddened B stars. An evolved sequence of $\mathrm{B} 3$ subgiants and giants terminating near $M_{V} \approx$ -3.5 or $V \approx 13.8$ is characteristic of what one would observe for a group of stars physically and coevally associated with GT Car (cf. Harris 1976, Group III).

In order to avoid undue confusion in the interpretation of Figure 6, the stars plotted are restricted to those identified as likely O- or B-type lying $\sim 0.05$ in $U-B$ above the intrinsic or reddened color-color relation, to a few faint blue stars not satisfying the above restriction, and to two red stars ( $G$ and 168 ) which have colors and magnitudes similar to GT Car. When dereddened to the supergiant relation of Fernie (1963), the color excesses derived for these last two stars are also similar to that predicted for GT Car. They are also the only red stars in the field for which this conclusion is valid, even though red stars populate the entire magnitude interval fainter than $V=10.8$.

The assumption that the 11 stars (nine B-type, two possible FG supergiants) identified by asterisks in Tables 1 and 5 form an association with GT Car is consistent with predictions from stellar evolutionary theory. A small group of associated KM supergiants with $B-V \approx 2.3$ and $V \approx 13$ should also be present in the field (cf. Mermilliod 1981) but would have been missed owing to the magnitude restrictions of the survey. It may be possible to search for such objects on the original plates. The mean reddening of the 11 potential companions to GT Car is $E_{B-V}(B 0)=0.72 \pm 0.02$ s.e., which corresponds to a space reddening for the Cepheid of $E_{B-V}=0.66 \pm 0.02$.

The ZAMS is plotted in Figure 6 for two cases corresponding to $E_{B-V}=0.05$ and $d=2.7 \mathrm{kpc}$ (the distance to the Car $\mathrm{OB} 1$ complex) and $E_{B-V}=0.72$ and $d=10.2 \mathrm{kpc}$ (the predicted distance to GT Car with the above reddening). The data in the figure imply that B stars of the Car OB1 complex with 
TABLE 5

Photographic Data for Stars IN The Field of GT CarinaE

\begin{tabular}{|c|c|c|c|c|c|c|c|c|c|c|c|}
\hline Star & $V$ & $B-V$ & $U-B$ & Star & $V$ & $B-V$ & $U-B$ & Star & $V$ & $B-V$ & $U-B$ \\
\hline $1 \ldots$ & 13.71 & 0.33 & 0.27 & $57 \ldots \ldots$ & 13.51 & 0.42 & 0.16 & 113 & 14.06 & 0.69 & 0.38 \\
\hline $2 \ldots \ldots$ & 13.76 & 0.53 & 0.06 & $58 \ldots \ldots$ & 14.14 & 0.10 & 0.10 & $114 \ldots \ldots$ & 14.40 & 0.45 & 0.15 \\
\hline $3 \ldots$ & 12.60 & 1.37 & 1.17 & $59 \ldots \ldots$ & 13.27 & 0.09 & 0.16 & $115 \ldots$ & 14.08 & 0.05 & -0.02 \\
\hline $4 \ldots \ldots$ & 12.74 & 0.27 & -0.24 & $* 60 \ldots \ldots$ & 14.09 & 0.55 & -0.07 & $* 116 \ldots \ldots$ & 14.36 & 0.52 & -0.09 \\
\hline $5 \ldots \ldots$ & 13.73 & 0.46 & 0.01 & $61 \ldots$ & 13.53 & 0.45 & 0.03 & $117 \ldots$ & 12.28 & 0.45 & 0.31 \\
\hline $6 \ldots \ldots$ & 14.27 & 0.61 & 0.18 & $62 \ldots \ldots$ & 13.86 & 0.28 & 0.04 & $118 \ldots \ldots$ & 13.85 & 0.35 & 0.24 \\
\hline $7 \ldots$ & 13.85 & 0.39 & 0.15 & $63 \ldots \ldots$ & 14.45 & 0.35 & -0.35 & $119 \ldots \ldots$ & 14.41 & 0.54 & 0.02 \\
\hline $8 \ldots \ldots$ & 13.52 & 0.16 & -0.33 & $* 64 \ldots \ldots$ & 14.11 & 0.53 & -0.18 & $120 \ldots \ldots$ & 14.28 & 0.77 & 0.16 \\
\hline $9 \ldots$ & 14.28 & 0.32 & 0.09 & $* 65 \ldots \ldots$ & 13.87 & 0.41 & -0.40 & $121 \ldots \ldots$ & 14.13 & 0.53 & 0.04 \\
\hline $10 \ldots$ & 13.97 & 0.48 & 0.23 & *66 .... & 14.06 & 0.52 & -0.42 & $122 \ldots \ldots$ & 11.72 & 1.75 & 1.59 \\
\hline $11 \ldots$ & 12.99 & 0.12 & -0.14 & $67 \ldots$ & 13.84 & 0.58 & 0.07 & $123 \ldots \ldots$ & 14.34 & 0.35 & 0.04 \\
\hline $12 \ldots \ldots$ & 14.20 & 0.33 & 0.14 & *68 .... & 13.84 & 0.53 & -0.06 & $124 \ldots \ldots$ & 14.33 & 0.58 & 0.04 \\
\hline $13 \ldots \ldots$ & 13.46 & -0.01 & 0.16 & $69 \ldots \ldots$ & 12.65 & 0.20 & -0.37 & $125 \ldots \ldots$ & 14.17 & 0.36 & -0.10 \\
\hline $14 \ldots \ldots$ & 14.07 & 0.46 & 0.06 & $70 \ldots$ & 12.83 & 0.28 & 0.25 & $126 \ldots \ldots$ & 13.60 & 0.01 & 0.15 \\
\hline $15 \ldots \ldots$ & 13.98 & 0.50 & 0.23 & $71 \ldots$ & 14.21 & 0.48 & 0.31 & $127 \ldots \ldots$ & 13.93 & 0.47 & 0.04 \\
\hline $16 \ldots$ & 13.39 & 0.95 & 0.65 & $72 \ldots \ldots$ & 13.58 & 0.49 & 0.03 & $128 \ldots \ldots$ & 13.45 & 0.02 & 0.01 \\
\hline $17 \ldots$ & 14.01 & 0.38 & 0.22 & $73 \ldots$ & 11.73 & 0.12 & -0.34 & $129 \ldots \ldots$ & 12.89 & 0.44 & 0.04 \\
\hline $18 \ldots \ldots$ & 12.93 & 0.07 & 0.04 & $74 \ldots \ldots$ & 12.47 & 0.34 & -0.01 & $130 \ldots$ & 13.61 & 0.39 & -0.01 \\
\hline $19 \ldots$ & 13.37 & 0.54 & 0.20 & $75 \ldots$ & 10.99 & 0.21 & -0.77 & $131 \ldots \ldots$ & 13.51 & 0.20 & -0.03 \\
\hline $20 \ldots \ldots$ & 14.01 & 0.30 & 0.09 & $76 \ldots$ & 11.01 & 0.26 & -0.67 & $132 \ldots \ldots$ & 13.15 & 0.15 & 0.13 \\
\hline $21 \ldots \ldots$ & 13.69 & 0.61 & 0.38 & $77 \ldots$ & 12.23 & 0.15 & -0.22 & $133 \ldots \ldots$ & 13.95 & 0.74 & 0.10 \\
\hline $22 \ldots \ldots$ & 12.89 & 0.22 & -0.05 & $78 \ldots \ldots$ & 13.35 & 0.15 & 0.04 & $134 \ldots \ldots$ & 13.18 & 0.44 & 0.25 \\
\hline $23 \ldots \ldots$ & 13.87 & 0.47 & 0.10 & $79 \ldots \ldots$ & 13.85 & 0.32 & 0.17 & $135 \ldots \ldots$ & 14.30 & 0.39 & 0.09 \\
\hline $24 \ldots \ldots$ & 13.68 & 0.49 & -0.03 & $80 \ldots \ldots$ & 12.22 & 0.42 & 0.31 & $136 \ldots \ldots$ & 13.78 & 0.17 & -0.22 \\
\hline $25 \ldots \ldots$ & 14.39 & 0.29 & 0.07 & $81 \ldots$ & 13.48 & 0.43 & -0.03 & *137 .... & 13.29 & 0.61 & -0.20 \\
\hline $26 \ldots \ldots$ & 13.21 & 0.34 & 0.10 & $* 82 \ldots \ldots$ & 13.69 & 0.50 & -0.10 & $138 \ldots \ldots$ & 12.48 & 0.90 & 0.59 \\
\hline $27 \ldots \ldots$ & 13.20 & 0.04 & 0.08 & $83 \ldots \ldots$ & 14.26 & 0.40 & 0.15 & $139 \ldots \ldots$ & 11.69 & 0.39 & 0.10 \\
\hline $28 \ldots \ldots$ & 11.40 & 1.44 & 1.57 & $84 \ldots \ldots$ & 12.67 & 0.15 & 0.05 & $140 \ldots \ldots$ & 14.40 & 0.40 & 0.09 \\
\hline $29 \ldots \ldots$ & 13.48 & 0.12 & -0.29 & $85 \ldots \ldots$ & 14.27 & 0.36 & -0.03 & $141 \ldots \ldots$ & 12.67 & 0.29 & -0.01 \\
\hline $30 \ldots \ldots$ & 12.37 & 0.24 & -0.38 & $86 \ldots \ldots$ & 14.41 & 0.31 & -0.14 & $142 \ldots \ldots$ & 14.15 & 0.45 & 0.22 \\
\hline $31 \ldots$ & 13.17 & 0.03 & 0.34 & $87 \ldots \ldots$ & 14.04 & 0.33 & 0.21 & $143 \ldots \ldots$ & 13.82 & 0.17 & -0.13 \\
\hline $32 \ldots \ldots$ & 13.22 & -0.09 & -0.16 & $88 \ldots \ldots$ & 11.91 & 0.01 & -0.05 & $144 \ldots \ldots$ & 13.26 & 0.27 & 0.01 \\
\hline $33 \ldots \ldots$ & 14.18 & 0.27 & 0.06 & $89 \ldots \ldots$ & 12.32 & -0.04 & 0.15 & $145 \ldots \ldots$ & 13.82 & 0.36 & 0.13 \\
\hline $34 \ldots \ldots$ & 14.21 & 0.12 & 0.22 & $90 \ldots \ldots$ & 12.00 & 0.74 & 0.69 & $146 \ldots$ & 14.02 & 0.76 & 0.37 \\
\hline $35 \ldots$ & 13.95 & 0.63 & 0.27 & $91 \ldots \ldots$ & 14.21 & 0.60 & 0.09 & $147 \ldots \ldots$ & 13.62 & 0.59 & 0.22 \\
\hline $36 \ldots \ldots$ & 14.25 & 0.44 & -0.05 & $92 \ldots \ldots$ & 14.19 & 0.46 & 0.04 & $148 \ldots \ldots$ & 10.92 & 0.13 & -0.12 \\
\hline $37 \ldots$ & 11.34 & 0.67 & 0.45 & $93 \ldots \ldots$ & 13.21 & 0.33 & 0.00 & $149 \ldots \ldots$ & 14.36 & 0.44 & 0.13 \\
\hline $38 \ldots \ldots$ & 13.91 & 0.68 & 0.30 & $94 \ldots \ldots$ & 11.03 & 0.42 & -0.03 & $150 \ldots \ldots$ & 13.97 & 0.23 & 0.06 \\
\hline $39 \ldots$. & 12.56 & 0.10 & 0.10 & $95 \ldots \ldots$ & 13.64 & 0.62 & 0.12 & $151 \ldots$ & 11.78 & 0.55 & 0.35 \\
\hline $40 \ldots \ldots$ & 12.46 & 0.17 & 0.14 & $96 \ldots \ldots$ & 12.89 & 0.63 & 0.20 & $152 \ldots \ldots$ & 12.12 & 1.67 & 1.55 \\
\hline $41 \ldots$ & 10.81 & 1.43 & 1.20 & $97 \ldots \ldots$ & 13.44 & 0.52 & 0.03 & $153 \ldots$ & 10.40 & 0.60 & -0.01 \\
\hline $42 \ldots \ldots$ & 12.92 & 0.49 & 0.04 & $98 \ldots \ldots$ & 12.75 & 0.01 & -0.44 & $154 \ldots \ldots$ & 13.07 & 0.24 & 0.13 \\
\hline $43 \ldots$ & 12.85 & 0.04 & -0.04 & $99 \ldots$ & 14.13 & 0.48 & 0.05 & $155 \ldots$ & 14.09 & 0.20 & 0.24 \\
\hline $44 \ldots \ldots$ & 12.36 & 0.37 & 0.13 & $100 \ldots \ldots$ & 13.96 & 0.47 & 0.10 & $156 \ldots \ldots$ & 14.36 & 0.66 & 0.09 \\
\hline $45 \ldots \ldots$ & 12.89 & 0.43 & 0.43 & $101 \ldots$ & 13.07 & 0.23 & -0.02 & $157 \ldots$ & 10.94 & 0.09 & -0.70 \\
\hline $46 \ldots \ldots$ & 12.72 & 0.54 & 0.04 & $102 \ldots \ldots$ & 14.03 & 0.57 & 0.06 & $158 \ldots \ldots$ & 11.09 & 0.05 & -0.75 \\
\hline $47 \ldots \ldots$ & 11.46 & 1.28 & 0.78 & $103 \ldots \ldots$ & 13.29 & 0.38 & 0.19 & $159 \ldots \ldots$ & 12.67 & 0.13 & 0.19 \\
\hline$* 48 \ldots \ldots$ & 14.14 & 0.49 & -0.31 & $104 \ldots \ldots$ & 13.87 & 0.17 & -0.11 & $160 \ldots \ldots$ & 12.72 & 1.10 & 0.86 \\
\hline $49 \ldots \ldots$ & 12.43 & 1.13 & 0.87 & $105 \ldots$ & 13.41 & 0.41 & 0.18 & $161 \ldots \ldots$ & 10.80 & 1.23 & 1.31 \\
\hline $50 \ldots \ldots$ & 13.33 & 0.45 & 0.17 & $106 \ldots \ldots$ & 14.42 & 0.79 & 0.07 & $162 \ldots \ldots$ & 12.45 & 0.39 & 0.14 \\
\hline $51 \ldots \ldots$ & 13.90 & 0.27 & -0.23 & $107 \ldots$ & 13.89 & 0.62 & 0.32 & $163 \ldots$ & 13.77 & 0.52 & 0.07 \\
\hline $52 \ldots \ldots$ & 13.96 & 0.38 & 0.04 & $108 \ldots$ & 13.73 & 0.40 & 0.22 & $164 \ldots \ldots$ & 13.61 & 0.47 & -0.01 \\
\hline $53 \ldots \ldots$ & 12.75 & -0.07 & 0.16 & $109 \ldots$ & 11.50 & 0.34 & 0.00 & $165 \ldots \ldots$ & 14.42 & 0.37 & 0.08 \\
\hline $54 \ldots \ldots$ & 13.59 & -0.06 & -0.31 & $110 \ldots$ & 11.95 & 1.33 & 1.44 & $166 \ldots \ldots$ & 14.39 & 0.59 & 0.04 \\
\hline $55 \ldots \ldots$ & 14.53 & 0.19 & -0.22 & $111 \ldots$ & 13.20 & 0.00 & 0.23 & $167 \ldots$ & 14.01 & 0.27 & -0.15 \\
\hline $56 \ldots \ldots$ & 13.41 & -0.03 & -0.28 & $112 \ldots$ & 13.95 & 0.24 & 0.04 & $* 168 \ldots \ldots$ & 13.31 & 1.14 & 0.86 \\
\hline
\end{tabular}

minimum foreground reddening are encountered throughout this field. The small amount of observed differential reddening is not detected visually from variations in star densities and must arise within the Carina spiral arm itself, which in this direction is being viewed almost tangentially. The detection of candidate stars associated with GT Car suggests that the line of sight passes through a relatively clear zone on the inside edge (or far side) of the Carina arm and intercepts a portion of the next outer arm (the local arm) $\sim 10 \mathrm{kpc}$ distant, as expected from the $21 \mathrm{~cm}$ picture of spiral structure in this region of the Galaxy. The relative transparency of the GT Car field may be due to the warping of the Galactic disk (and, hence, much of 


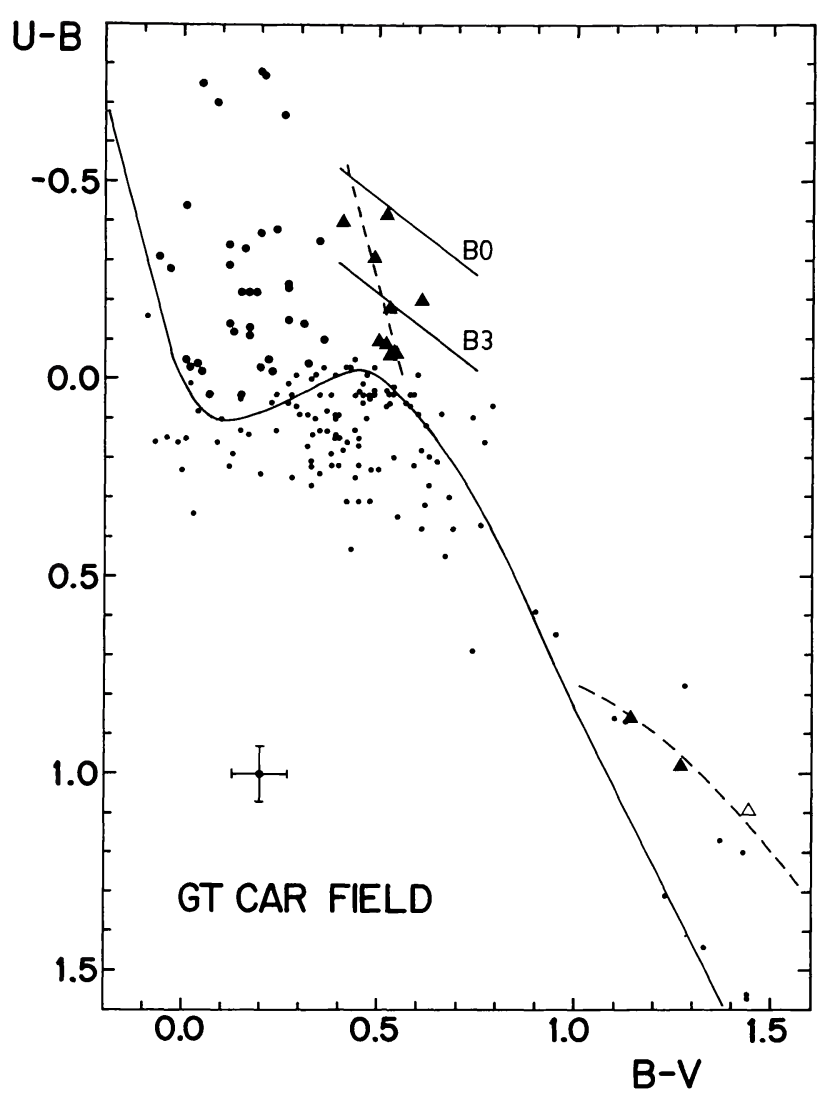

FIG. 5.-Color-color plot for stars in the GT Car field. The solid line is the intrinsic relation for dwarfs, and dashed lines depict the B dwarf and FG supergiant relations reddened by the amount predicted for GT Car. The symbols depict photometrically identified B stars (large dots), other stars in the field (small dots), potential companions to GT Car (filled triangles), and GT Car at mean light (open triangle).

the reddening material) to more negative latitudes along this line of sight.

Follow-up work is clearly needed. Photoelectric and spectroscopic observations of the 11 candidate stars in our putative GT Car association are needed to confirm their photometric identifications. Additionally, association stars cannot be used to establish an accurate distance to the Cepheid without reliable estimates of their absolute magnitudes inferred from MK spectral classifications and/or $\mathrm{H} \beta$ photometry, and radial velocities to test their physical association with GT Car. About 3' west-southwest of GT Car is a compact ( $\sim 0.5$ diameter $)$ anonymous cluster of faint stars which might also be associated with the Cepheid. Bright cluster stars were not measured here owing to crowding problems but should be resolvable with $\mathrm{CCD}$ imaging.

\section{3. $W Z$ Sagittarii}

WZ Sgr is a 21.850 Cepheid with $\langle V\rangle=8.023$ and $\langle B\rangle-$ $\langle V\rangle=1.404$ (Caldwell \& Coulson 1987), located at galactic coordinates $l=12.11, b=-1.31$. It lies on the edge of a prominent dust lane which runs through the Sagittarius sec- tion of the Milky Way, in a field which is moderately rich in open clusters and young OB stars. Among the recognized associations in this field are Sgr OB1 and Sgr OB4 at distances of 1.8 and $2.4 \mathrm{kpc}$, respectively (Humphreys 1978), and Sgr R1, a loose group of B stars illuminating reflection nebulosity 1.2 kpc distant (Racine 1968). Fernie (1962) first drew attention to the possibility that WZ Sgr might be a member of Sgr OB4, although it was later argued by Tzarevsky (1970) from proper motion data that membership in Sgr OB1 was more likely. Published radial velocities for the OB stars appear unable to resolve the issue since they exhibit rather large scatter, which makes the discrimination of group velocities difficult at this longitude. In addition, an evolutionary age of $\sim 3.0 \times 10^{7} \mathrm{yr}$ predicted for a $22^{\mathrm{d}}$ classical Cepheid (e.g., Kippenhahn \& Smith 1969) implies an earliest spectral type of B2.5-B3 for similarly aged, main-sequence companions, thereby eliminating a possible association of WZ Sgr with bright OB stars in its field.

Alternatively, Turner (1984) noted that several of the nearest optical companions to WZ Sgr are reddened mid-to-late B-type stars which could constitute the brightest members of a sparse cluster containing the Cepheid. Star counts are of limited value for delineating this cluster owing to the extremely patchy nature of the visible dust obscuration in the field. A slight increase in star densities centered 3'6 northwest of WZ Sgr was suggested by Turner (1984) to coincide with the sparse cluster core. Curiously, the sparse clump of faint stars centered only $1^{\prime}$ east of WZ Sgr (see Fig. 1 of Turner 1984) was not identified as a region of enhanced star density. Nevertheless, the detection of several B-type stars near WZ Sgr in a restricted photoelectric survey was a promising start to the more extensive photographic search presented here.

Table 2 presents the photoelectric sequence and Table 6 the 268 photographically-measured stars in the field of WZ Sgr,

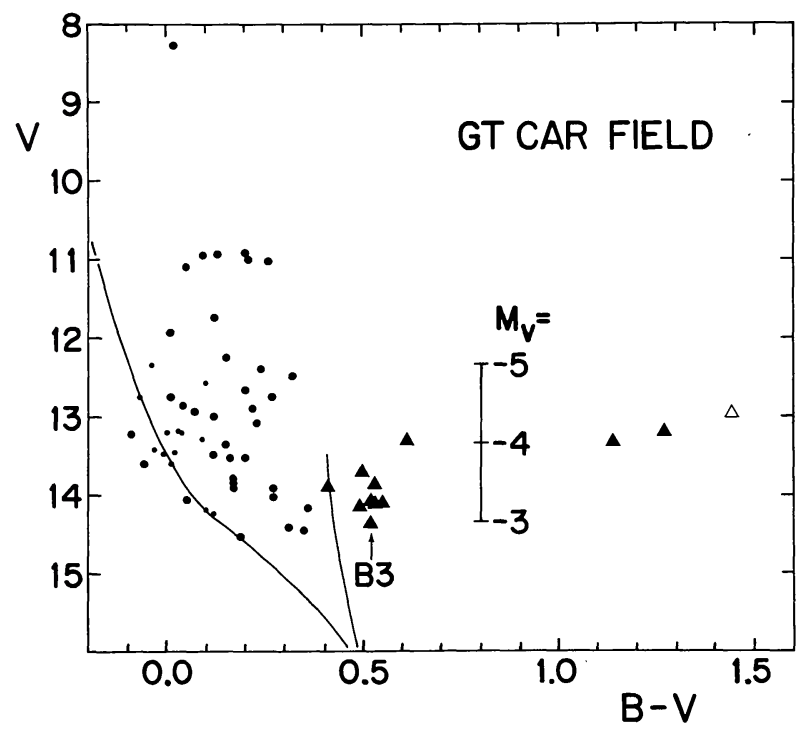

FIG. 6.-Color-magnitude diagram for stars in the GT Car field, with symbols as in Fig. 5. Solid lines depict the ZAMS for $E_{B-V}=0.05$ and $d=$ $2.7 \mathrm{kpc}($ left $)$, and $E_{B-V}=0.72$ and $d=10.2 \mathrm{kpc}$ (right). 


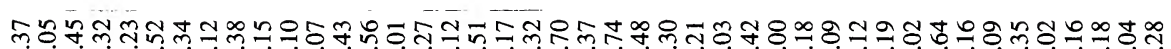

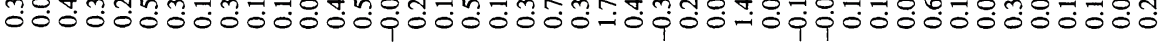

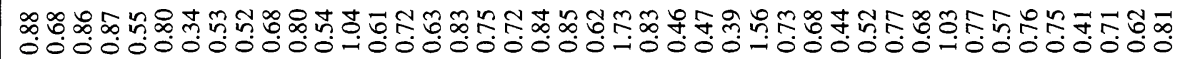

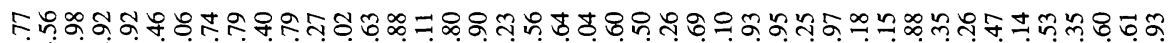

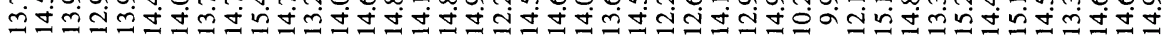

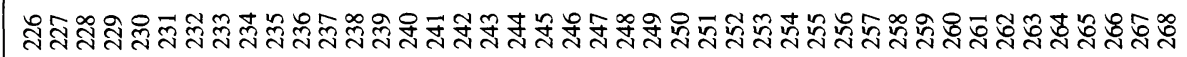

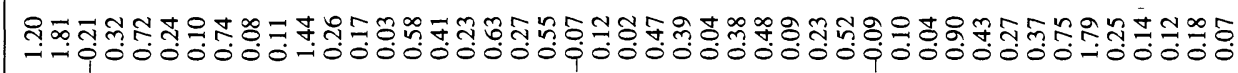

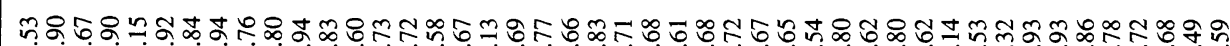
-

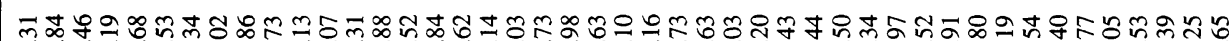

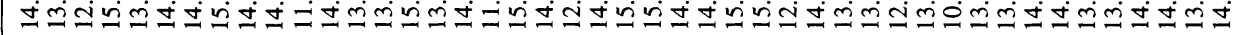

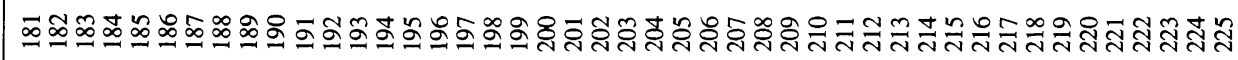

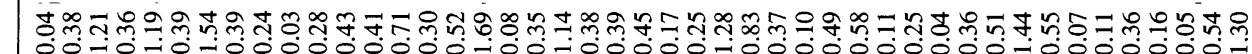

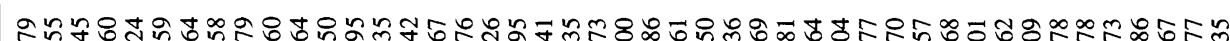

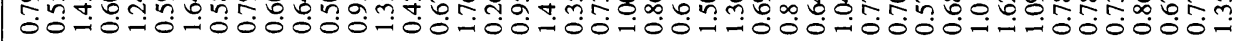

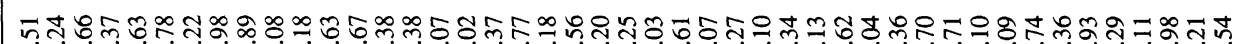

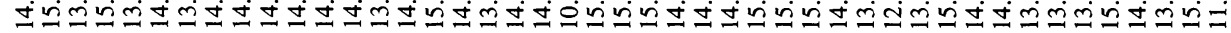

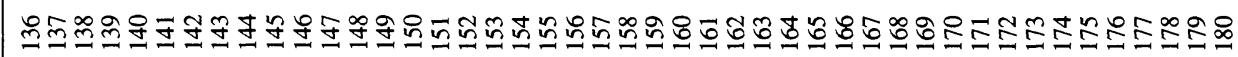
*

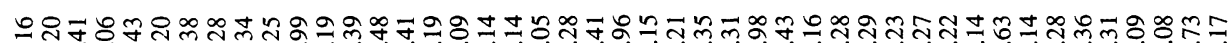
- op

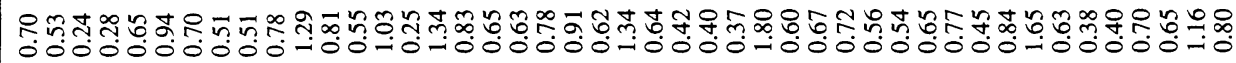

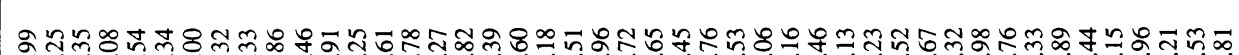

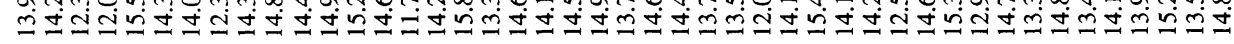

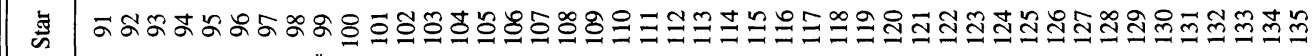

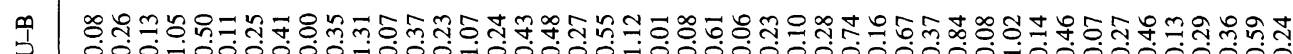
- 0 0.90000,0000-0000000000

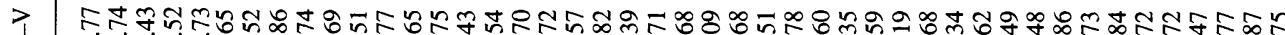

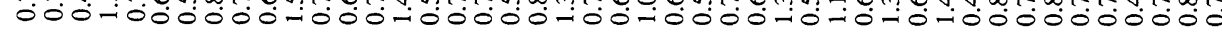

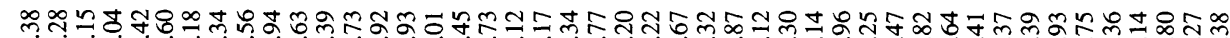

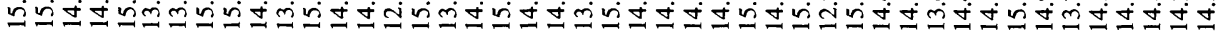

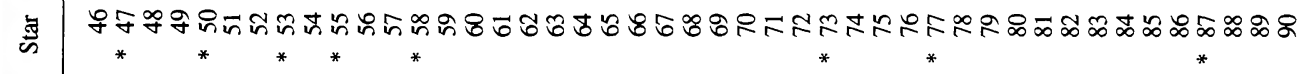

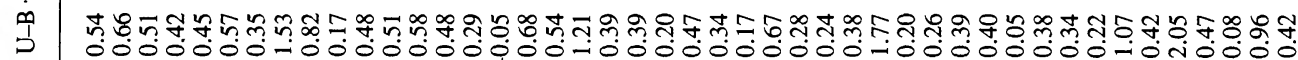

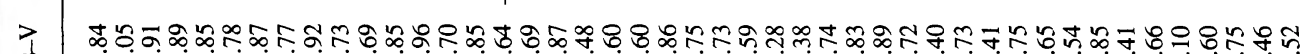

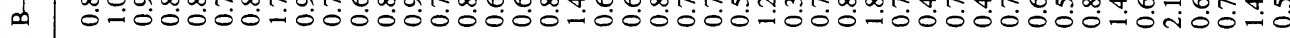

$>$ 궁

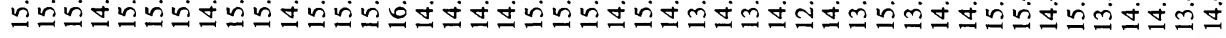

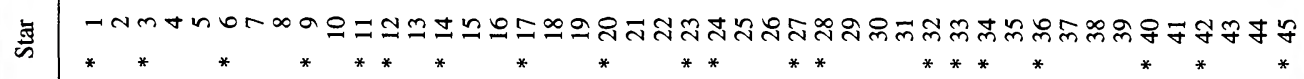


with the stars identified in Figure 7 (Plate 3 ). The survey area concentrates on two circular regions which overlap near WZ Sgr. The region northwest of the Cepheid was chosen to encompass the region of the proposed cluster found in the star counts, while the larger region east of the Cepheid was selected because its apparent excess of bright stars is similar in appearance to a loose cluster (which it proves to be). There are a number of other star concentrations $15^{\prime}-20^{\prime}$ distant from WZ Sgr which also give the appearance of loose, unstudied clusters, but these were left for later examination. Special efforts were made to measure many of the faint stars concentrated $\sim 1$ ' east of WZ Sgr when it became apparent from measures of the brighter stars that these objects might constitute a physical cluster. The photometric data confirm the visual impression that there is a sparse cluster centered at this point, but it is heavily masked and reddened by intervening dust clouds. Conversely, the increase in star counts northwest of the Cepheid can be traced to gaps in the veiling dust rather than to a suspected star cluster.

Figure 8 is a color-color diagram for the data of Tables 2 and 6. Owing to the dominating effects of differential reddening in the field of WZ Sgr, the stars were analyzed by the variableextinction method using a derived reddening line for the region of slope $E_{U-B} / E_{B-V}=0.78$ (see Turner 1989). All stars were dereddened individually to the intrinsic relation for

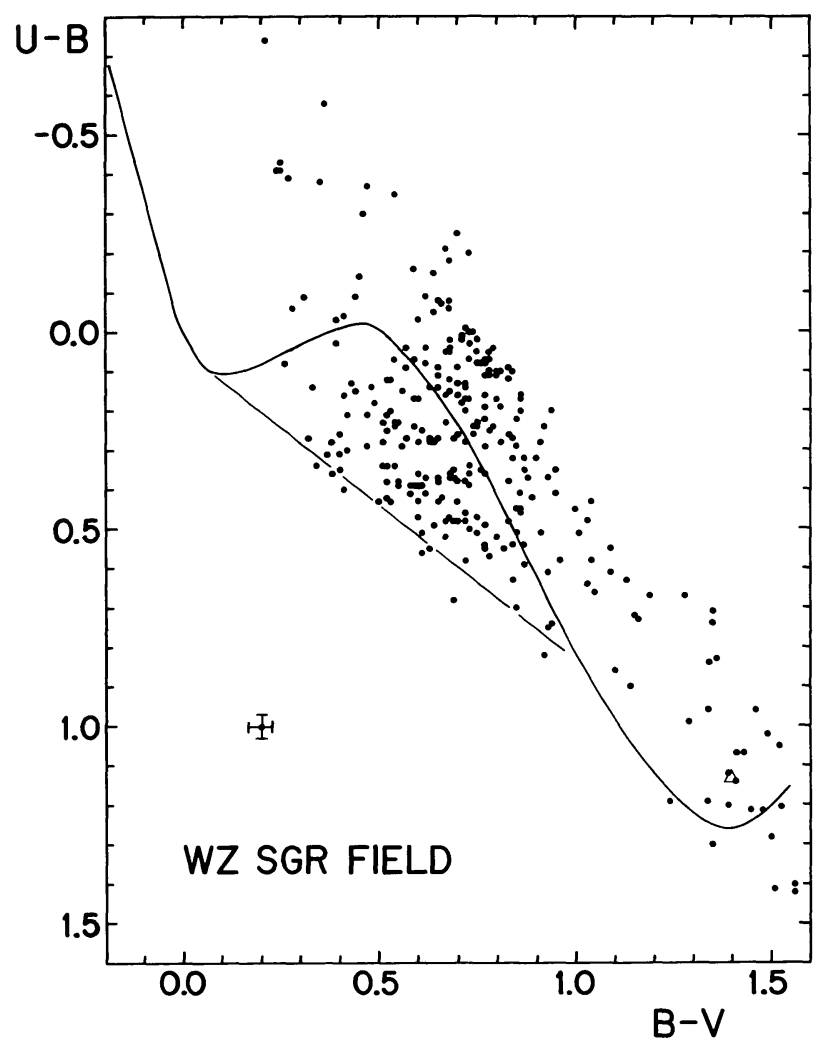

FIG. 8.-Color-color plot for stars in the WZ Sgr field. The solid curve is the intrinsic relation for dwarfs, and a line depicts the reddening relation for A2 stars. An open triangle represents the colors of WZ Sgr at mean light. dwarfs, noting multiple solutions as they arose, with derived color excesses adjusted to those appropriate for a B0 star (Fernie 1963). An iterative procedure was used, in conjunction with the visibility of extremely faint stars on a copy of the POSS $B$ plate of the field, to spatially map the reddening near WZ Sgr. Color excesses for stars with unambiguous reddening solutions correlate directly with the foreground dust obscuration on the POSS, a result which guided the selection of optimum values for stars with ambiguous reddening solutions. The visibility of faint stars on the POSS also helped to fill in gaps in the reddening map imposed by the irregular distribution of reddened stars across the field. The resulting space reddening plot is given in Figure 9, from which it is evident that dense portions of the dust in the field of WZ Sgr appear as narrow strands of large reddening running in a direction roughly parallel with Gould's Belt. Areas of low reddening correspond to thinner portions of the dust clouds through which the diffuse glow of the Milky Way's stellar background is more easily detected.

The data of Figure 8 emphasize the general shortage of unreddened objects in this field. A minimum reddening of $E_{B-V} \approx 0.35$ applies to all stars of types $\mathrm{B}, \mathrm{A}$, and $\mathrm{F}$, and most of types $\mathrm{K}$ and $\mathrm{M}$ (which are probably giants), while many of the stars lying close to the intrinsic relation for $\mathrm{G}$ dwarfs could be reddened stars of earlier type. Extinction versus distance diagrams by Neckel \& Klare (1980) for the fields surrounding WZ Sgr demonstrate that the reddening originates in dust clouds lying about 300-500 pc distant. The distance moduli of the few program stars assumed here to be unreddened $G$ dwarfs are less than $V_{0}-M_{V} \approx 8.25$, which indicates a distance of $\sim 450 \mathrm{pc}$ for the dust clouds. Additional confirmation for these estimates is found in the low star densities derived from the POSS $B$ plates for the more opaque regions south and east of WZ Sgr.

Figure 10 is a variable-extinction diagram for stars in the WZ Sgr field based upon ZAMS estimates of absolute magnitude (invariably underestimates of the true luminosities for many stars). A lower envelope sequence of points of slope $R=$ $A_{V} / E_{B-V} \approx 3$ is evident in the data, with stars representing this lower envelope concentrated in the region of photoelectric standard B. Stars near WZ Sgr exhibit a trend towards a similar $R \approx 3$ solution displaced to systematically smaller values of $V-M_{V}$. Complete samples of these two distinct lower envelope sequences were analyzed using regression and nonparametric fits of straight lines to the data. A value of $R=3.09 \pm$ 0.18 was obtained for stars associated with star $\mathrm{B}$, and $R=$ $2.93 \pm 0.08$ was found for stars associated with WZ Sgr. A best estimate of $R=3.0( \pm 0.1)$ was assumed to apply to the dust extinction in this region.

$R=3.0$ relations are plotted in Figure 10 for the intrinsic distance moduli of the separate lower envelope samples. The group associated with star B has $V_{0}-M_{V}=11.84 \pm 0.12$ s.d. ( distance $=2.33 \pm 0.13 \mathrm{kpc}$ ), while the group associated with WZ Sgr has $V_{0}-M_{V}=11.26 \pm 0.10$ s.d. (distance $=1.79 \pm$ $0.08 \mathrm{kpc}$ ). The more distant group lies at the same distance as Sgr OB4, while the closer group corresponds in distance to Sgr OB1. These two concentrations of B- and A-type stars also appear as distinct peaks in intrinsic distance modulus in Figure 11 , which plots star numbers versus reddening-corrected dis- 


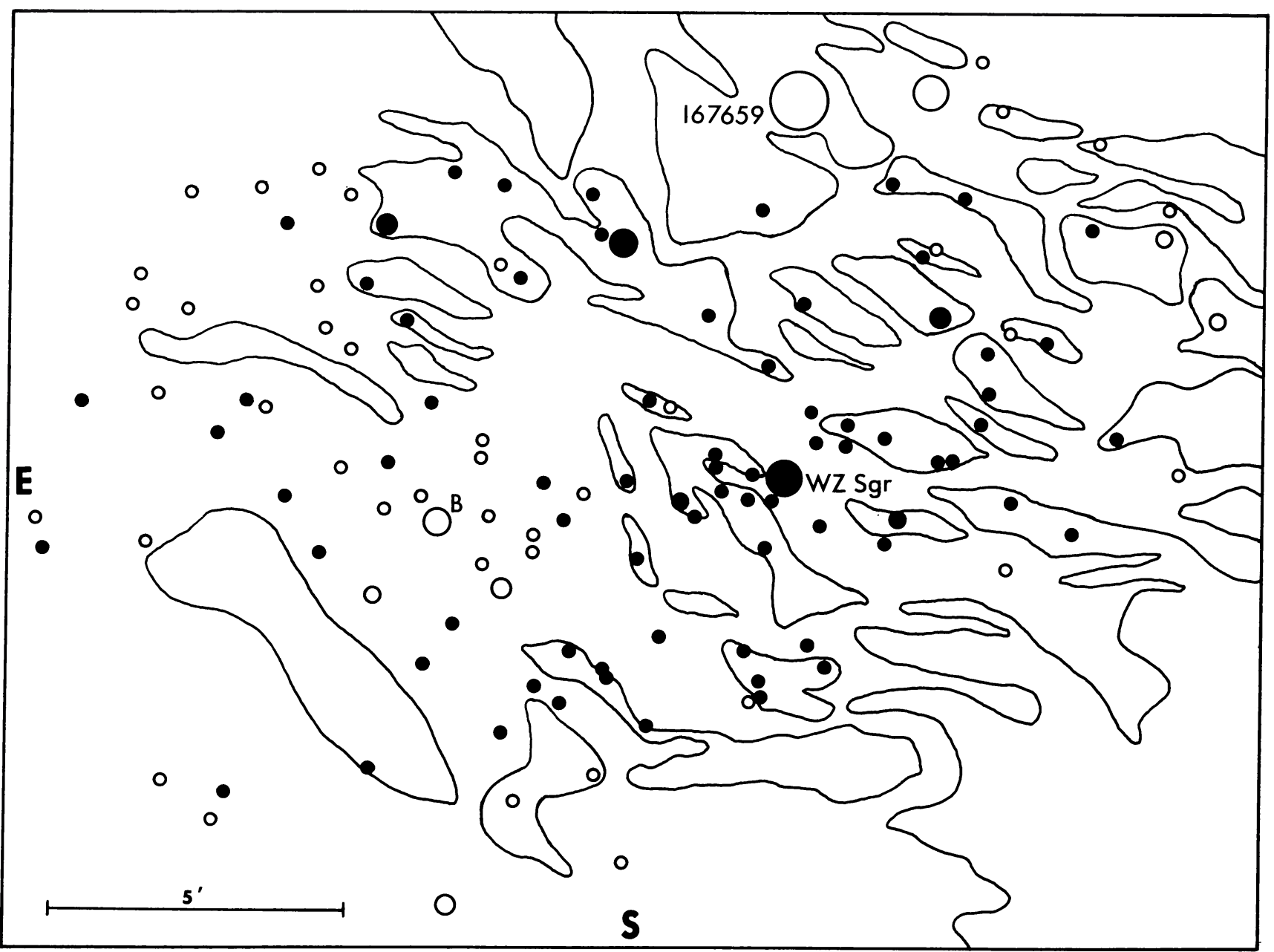

FIG. 9.- Space reddening plot for the WZ Sgr field. Likely members of the WZ Sgr cluster are plotted as filled circles, and stars likely to be associated with star B and Sgr OB4 are plotted as open circles. Lines enclose regions where the reddening is less than $E_{B-V}=0.60$.

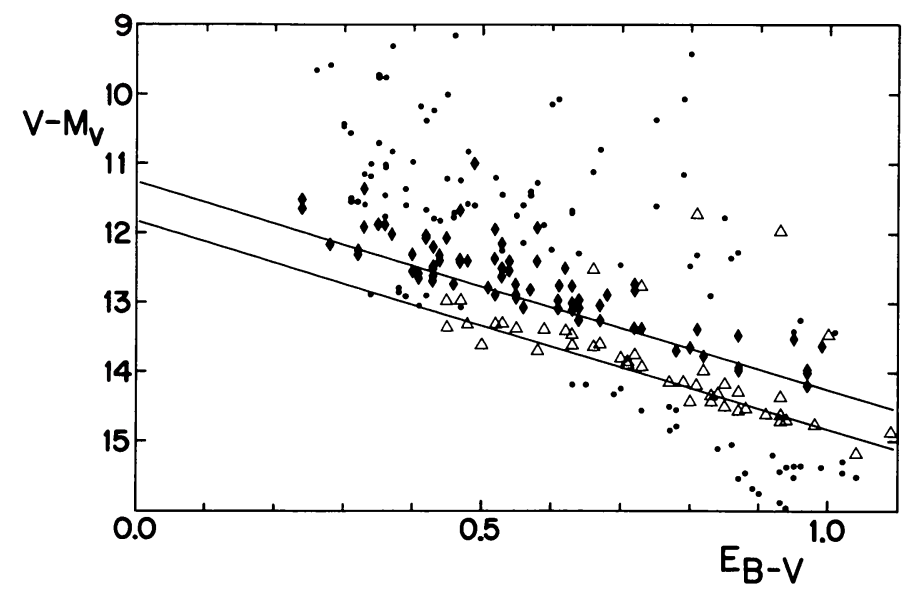

FIG. 10.- Variable-extinction diagram for stars in the WZ Sgr field. Likely members of the WZ Sgr cluster are represented by diamonds, and likely members of Sgr OB4 by open triangles. Small dots are other stars in the field. Lines of slope $R=3.0$ represent fits to the data.

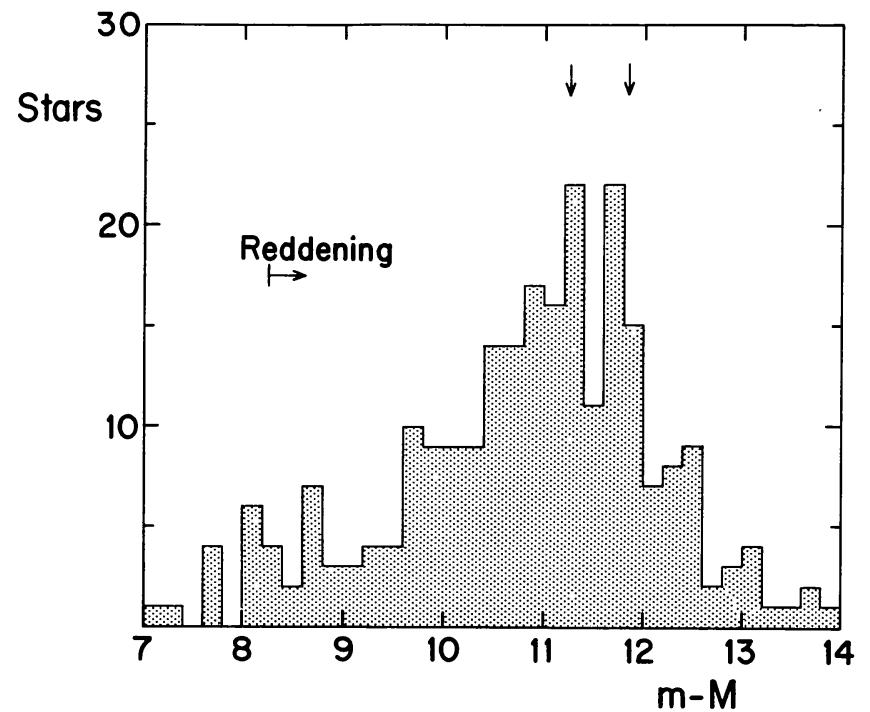

FIG. 11.-A histogram of the derived intrinsic distance moduli for stars plotted in Fig. 10. Arrows indicate the distance moduli for the two stellar groups identified in this paper. 
tance modulus in 0.2 mag bins. It is a reflection of the small size of the photometric errors that two groups in the same line of sight separated by only 0.6 mag in distance modulus can be detected in such a plot, particularly when the scatter toward systematically small values of $V-M_{V}$ is taken into account.

Likely members of the two groups of stars are identified by different symbols in Figures 9 and 10, with membership selection loosely based upon spatial location, distance modulus, and position in the group's color-magnitude diagram. The nearer group of stars (designated by asterisks in Tables 2 and 6 ) is concentrated toward WZ Sgr and forms a sparse cluster centered near star W, $0.5^{\prime}$ southeast of the Cepheid. The more distant group of stars contains members all over the field, but concentrates near star B. There is a suggestion of a separate concentration of the more distant group just off the edge of the field to the northeast. An anonymous cluster of 10th mag stars is visible here on the POSS, but a photometric study is needed to confirm its association with Sgr OB4.

IAU designations for open clusters are based upon their 1950 coordinates, to the nearest minute in right ascension and the nearest 0.1 in declination. Stars $W$ and $B$ are separated by only $5^{\prime}$ in right ascension and have identical rounded coordinates in this scheme. We have therefore designated the two newly discovered clusters as C1814-191a (the WZ Sgr cluster) and C1814-191b (the cluster near photoelectric standard B). Figure 12 is a reddening-corrected color-magnitude diagram for the 78 potential members of the WZ Sgr cluster, including the Cepheid. Figure 13 is a similar diagram for the 47 potential members of C1814-191b and Sgr OB4, with the subgroup of 22 stars lying within $5^{\prime}$ of star B identified by different symbols.

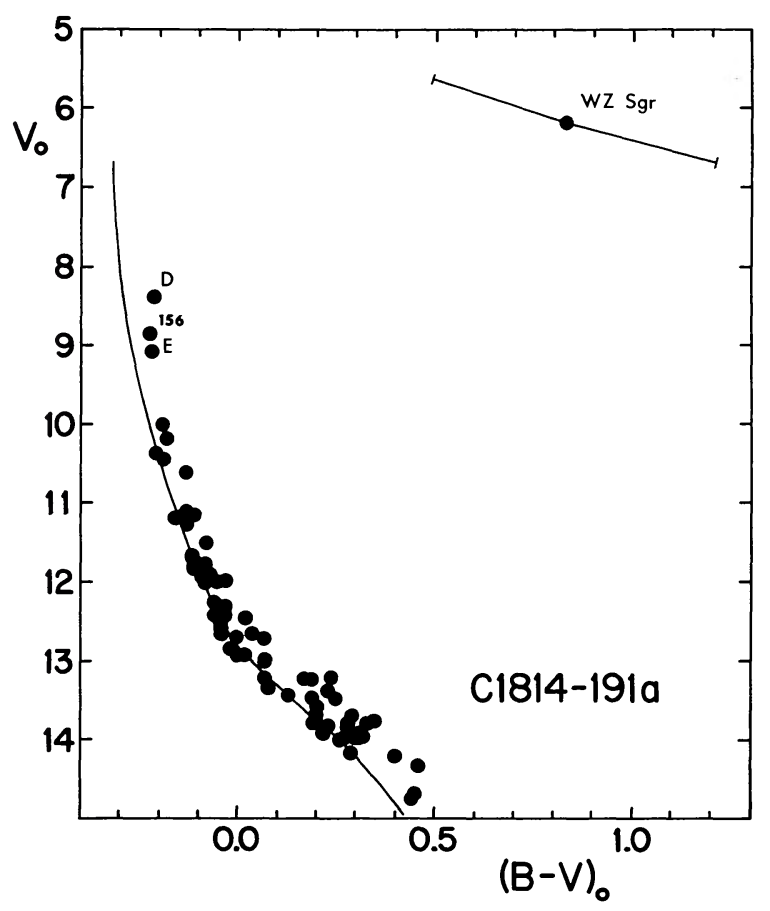

FIG. 12.-A reddening-free color-magnitude diagram for stars identified as possible members of the cluster associated with WZ Sgr. The ZAMS is plotted for $V_{0}-M_{V}=11.26$.

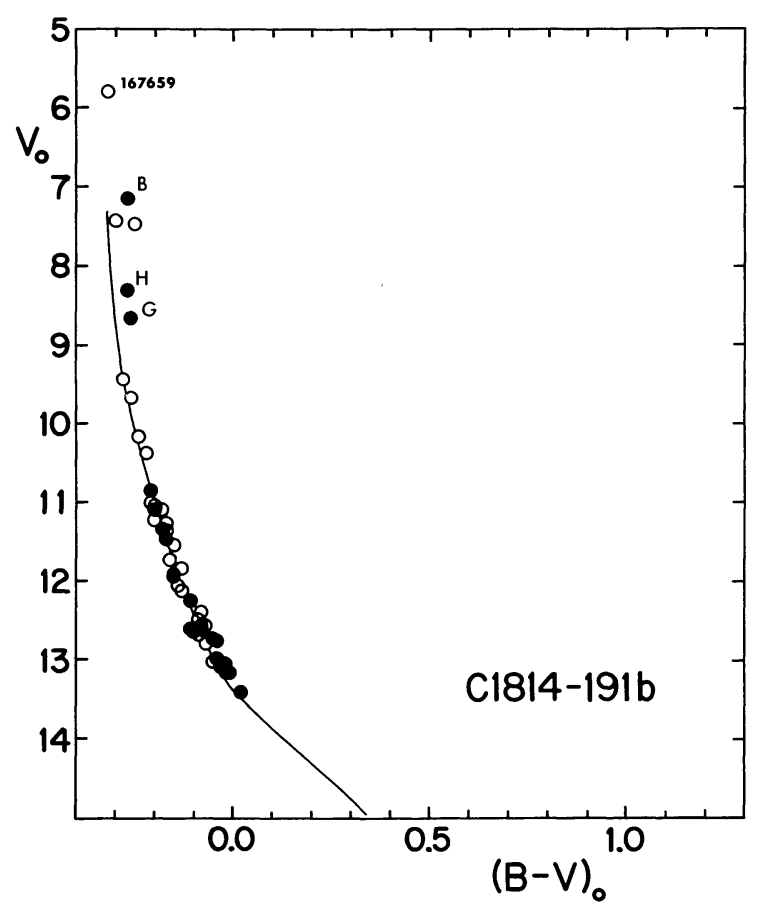

FIG. 13.-A reddening-free color-magnitude diagram for stars identified as likely members of Sgr OB4 (open circles) and the sparse cluster surrounding star B (filled circles). The ZAMS is plotted for $V_{0}-M_{V}=$ 11.84 .

The color-magnitude diagram of Figure 12 summarizes the available information on the dereddened parameters for possible members of the WZ Sgr cluster. The scatter above the ZAMS could be due to binarity and nonmember contamination, but even eliminating these particular stars from the sample does not affect the apparent concentration toward WZ Sgr. The cluster therefore is a true spatial concentration, despite the fact that the brightest members (including stars D, E, and 156) are located near the edge of the cluster field. Burki (1978) found similar properties for the massive members of several prominent young clusters, and Harris (1980) has speculated that this may be a characteristic of open clusters of all ages. Identified members of the WZ Sgr cluster are found over a field of almost $10 \mathrm{pc}$ (not an unreasonable diameter for a cluster of this type), so a few undiscovered bright cluster members may lie among the unsampled 10th mag stars southwest of the $\mathrm{Ce}$ pheid.

The spectroscopic observations for stars D and E (Table 7) are useful for testing the photometric results. Since the spectral classifications for both stars are quite close to their photometrically inferred types, and their radial velocities are the same as the observed systemic velocity for WZ Sgr (Caldwell \& Coulson 1987), there is clearly a good case for cluster membership of the Cepheid. Unfortunately, the errors in the velocities for the B stars are probably larger than the formal errors quoted in Table 7, owing to the low dispersion of the spectra and their relatively low signal-to-noise ratios of $\sim 30$ to 40 . Also, time limitations permitted only a single observation of each star. Additional spectroscopic observations for possible cluster stars 
TABLE 7

SPECTROSCOPIC OBSERVATIONS OF STARS NEAR WZ SAGITTARII

\begin{tabular}{cccccc}
\hline \multicolumn{1}{c}{ Star } & $\begin{array}{c}\text { HJD } \\
2,448,000+\end{array}$ & $\begin{array}{c}V_{R} \\
\left(\mathrm{~km} \mathrm{~s}^{-1}\right)\end{array}$ & $\begin{array}{c}V \text { sin } i \\
\left(\mathrm{~km} \mathrm{~s}^{-1}\right)\end{array}$ & $\begin{array}{c}\text { Spectral } \\
\text { Type }\end{array}$ & $\begin{array}{c}\text { Photometric } \\
\text { Type }\end{array}$ \\
\hline D $\ldots \ldots \ldots \ldots$ & 480.7499 & $-17 \pm 6$ & $156 \pm 22$ & B2-3 IV: & b2.5 iv \\
E $\ldots \ldots \ldots \ldots$ & 482.7299 & $-18 \pm 6$ & $203 \pm 7$ & B2 IV: & b2.5 iv-v \\
WZ Sgr $\ldots \ldots$ & $\ldots$ & $-17.8^{\mathrm{a}}$ & $\ldots$ & $\ldots$ & $\ldots$ \\
\hline
\end{tabular}

${ }^{a}$ From Caldwell \& Coulson 1987.

are therefore essential for strengthening the case for cluster membership of WZ Sgr.

It is of interest to note that the lower main-sequence of the WZ Sgr cluster terminates near $(B-V)_{0}=0.28$ in Figure 12, with a small gap of width $\sim 0.1 \mathrm{mag}$ in $(B-V)_{0}$ redward of this. This feature does not appear to be due to incomplete sampling of faint cluster stars. Such a gap characterizes the final stages of pre-main-sequence evolution (Ulrich 1971), when gravitational contraction is temporarily halted as ${ }^{3} \mathrm{He}$, mostly produced by earlier ${ }^{2} \mathrm{D}$ burning, is consumed by nuclear reactions in the stellar interior. A cluster turnon point at $(B-V)_{0}=0.28$ corresponds to a cluster age consistent with that derived from the observed turnoff point at $(B-V)_{0}=$ -0.22 (spectral type B2.5) for any reasonable initial abundance of ${ }^{3} \mathrm{He}$. Therefore, a deeper photometric search for additional main-sequence members of the cluster might be relatively unproductive.

The color-magnitude diagram for C1814-191b and Sgr OB4 (Fig. 13) is typical of a cluster younger in age than the WZ Sgr cluster, with stars belonging to Sgr OB4 apparently younger than stars in C1814-191b. A comparison with the models of Maeder \& Meynet (1988) results in age estimates of $\sim 4 \times 10^{6}$ $\mathrm{yr}$ for HD 167659 and star A, and $\sim 10^{7} \mathrm{yr}$ for luminous cluster members. It is not unusual to find subgroups of slightly different age in OB associations. However, there do not appear to be any stars older than $\sim 10^{7}$ yr among the $2.33 \mathrm{kpc}$ group, nor stars younger than $\sim 2.9 \times 10^{7} \mathrm{yr}$ among the $1.79 \mathrm{kpc}$ group. Based upon the age criterion, WZ Sgr cannot be physically associated with stars in Sgr OB4 and C1814-191b, yet is of comparable age to stars in its surrounding cluster. This is clearly a strong argument in favor of it being a cluster member.

The space reddening of WZ Sgr is $E_{B-V}(\mathrm{~B} 0)>0.6$ according to the results of Figure 9. The average reddening of the seven stars within $1^{\prime}$ of WZ Sgr is $\left\langle E_{B-V}\right\rangle=0.617 \pm 0.015$ s.e., while for the 22 stars within a larger $2^{\prime}$ radius it is $\left\langle E_{B-V}\right\rangle=0.600 \pm$ 0.022 s.e. The mean of these estimates, $E_{B-V}(\mathrm{~B} 0)=0.61 \pm$ 0.01 or $E_{B-V}($ Cepheid $)=0.56 \pm 0.01$, was adopted as the space reddening of WZ Sgr. It corresponds closely to photometric estimates, based upon six-color photometry, by Parsons \& Bouw (1971) and Parsons \& Bell (1975), which have been transformed to the system of Turner, Leonard, \& English (1987).

The parameters derived for WZ Sgr as a member of C1814$191 \mathrm{a}$ are $\left\langle M_{V}\right\rangle=-5.07 \pm 0.10$ and $(\langle B\rangle-\langle V\rangle)_{0}=0.84 \pm$ 0.01 . By way of comparison, the relations of van den Bergh (1977) predict values of $\left\langle M_{V}\right\rangle=-5.06 \pm 0.26$ and $(\langle B\rangle-$ $\langle V\rangle)_{0}=0.91 \pm 0.06$ for a classical Cepheid of identical period. The similarity of these estimates is a further argument for cluster membership, in addition to those based upon spatial coincidence, radial velocity, and cluster age. Additional spectroscopic observations, and possibly proper motion data, should establish an even stronger case for cluster membership of WZ Sgr.

\subsection{SW Velorum}

SW Vel is a 23.442 Cepheid with $\langle V\rangle=8.121$ and $\langle B\rangle-$ $\langle V\rangle=1.151$ (Caldwell \& Coulson 1987). It lies $3^{\circ}$ below the Galactic plane in Vela, within the faint outer filaments of the Gum nebula, only $15^{\prime}$ from the cluster NGC 2660 . A close coincidence in distance, reddening, and spatial location of SW Vel with NGC 2660 was remarked upon by Hartwick \& Hesser (1973) in their photometric study of this cluster, although possible cluster membership was not considered likely owing to the marked disparity in age between the two. NGC 2660 has a turnoff point age in excess of $10^{9} \mathrm{yr}$, compared to an expected age of $\sim 2.4 \pm 0.5 \times 10^{7} \mathrm{yr}$ for SW Vel based upon its period (e.g., Kippenhahn \& Smith 1969). A more promising connection was noted by Turner (1979), who found that the larger environs of SW Vel contain a number of OB stars which seem to have similar distances and ages to those predicted for the Cepheid. Additional considerations regarding this possibility were made by Eggen (1982) and Gieren (1988).

The present search encompasses stars lying within a $28^{\prime}$ diameter field centered on SW Vel, as shown in Figure 14 (Plate 4). Photographic $U B V$ data are presented in Table 8 for 397 stars, and $B V$ data for an additional 35. Although the field of SW Vel includes NGC 2660 and its rich supply of photoelectric standards (Hartwick \& Hesser 1973), the photographic measures were calibrated using only the Table 1 sequence. Stars 219 and 229 in Table 8 are identical to stars 9021 and 9014 (Z) of Hartwick \& Hesser, and the present data for these stars generally agree with their photoelectric values to within $\sim 0.02 \mathrm{mag}$ in magnitude and color. Test measures for a few other Hartwick \& Hesser standards produced no significant differences in derived magnitude or color which might indicate the presence of systematic errors in the present data set. Our data should therefore be fairly closely matched to the standard $U B V$ system.

Figure 15 is the color-color diagram for stars in the SW Vel field (Tables 1 and 8 ). A significant fraction of this sample can be identified as late B- and A-type stars reddened by $\sim 0.4$ in 


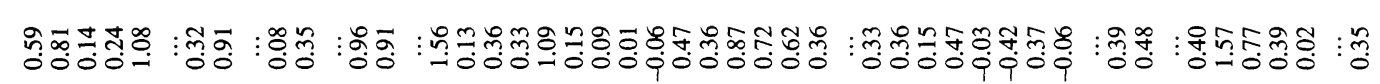

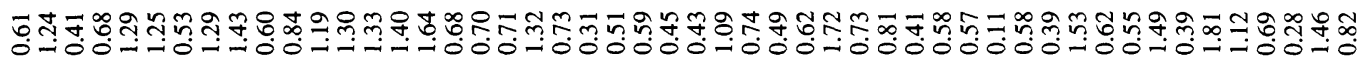

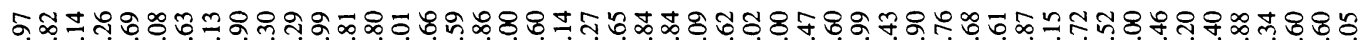

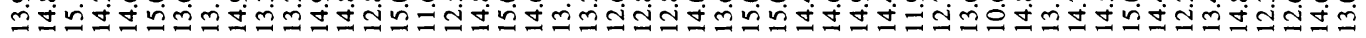

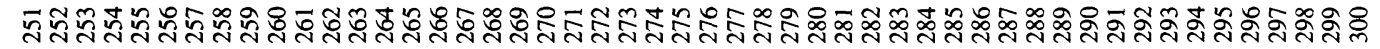

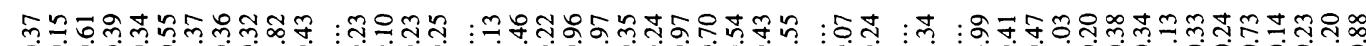
-0000000 00 0 000-000-00000-0

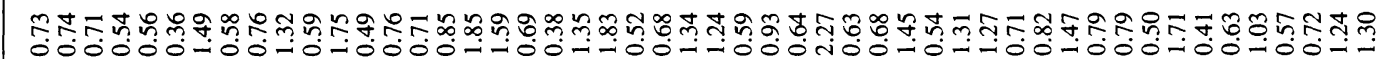

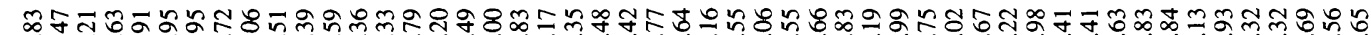

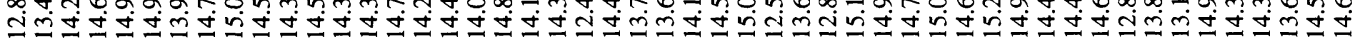

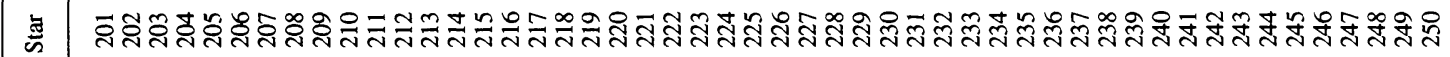

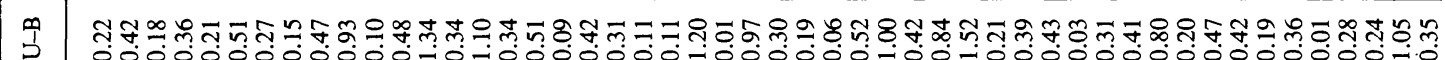

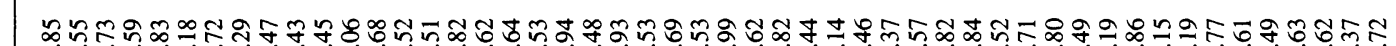
d 0.0000000

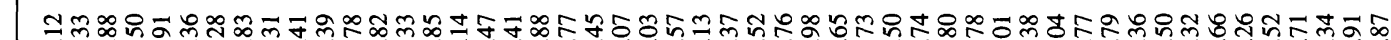

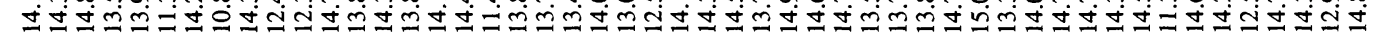

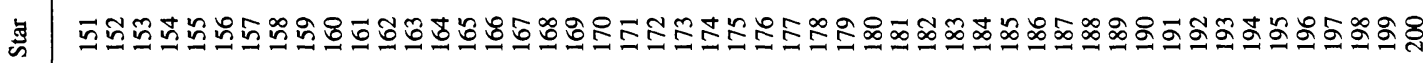

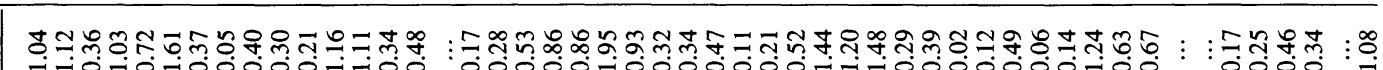

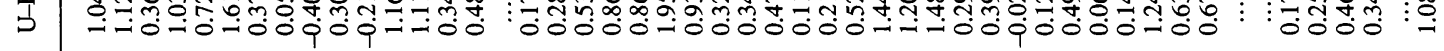

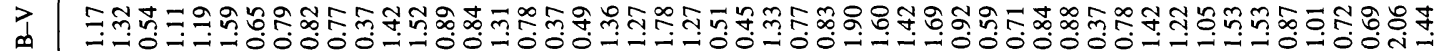

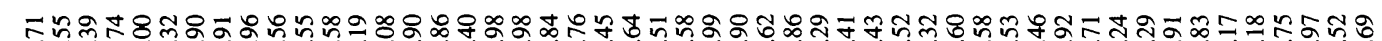

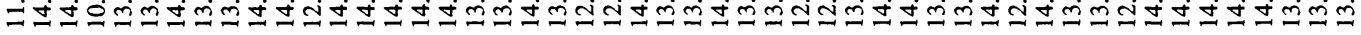

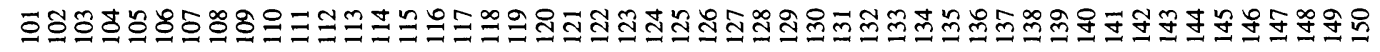

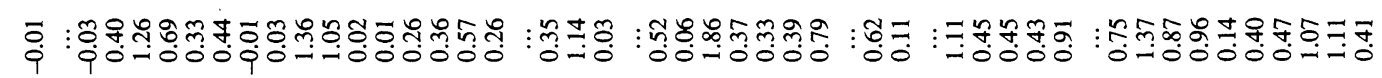

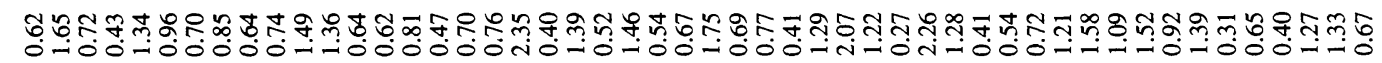

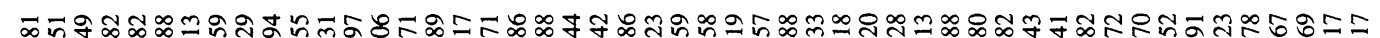

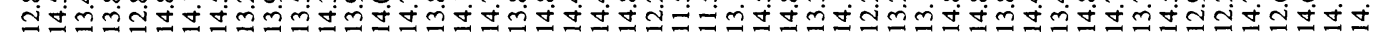

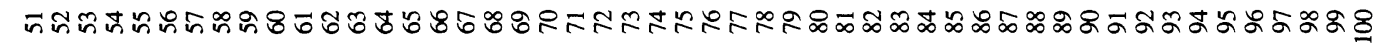

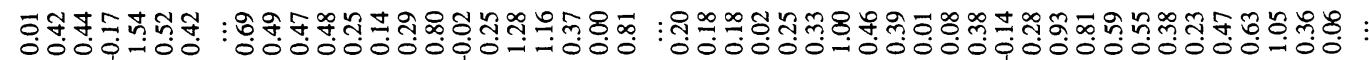

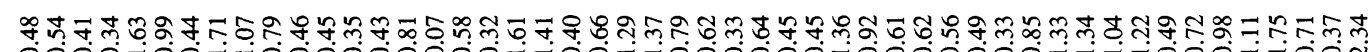

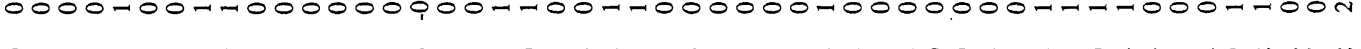

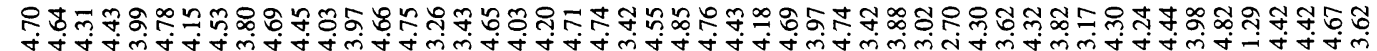

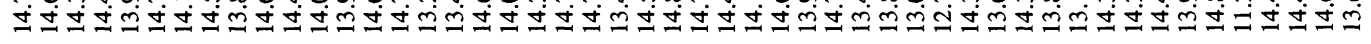
忽 ー 
TABLE 8-Continued

\begin{tabular}{|c|c|c|c|c|c|c|c|c|c|c|c|}
\hline Star & V & $B-V$ & U-B & Star & V & B-V & U-B & Star & V & B-V & U-B \\
\hline 301 & 13.33 & 0.36 & 0.39 & 345 & 14.80 & 0.62 & 0.39 & 389 & 13.88 & 0.75 & 0.32 \\
\hline 302 & 14.27 & 0.70 & 0.23 & 346 & 14.44 & 0.82 & 0.19 & 390 & 14.28 & 0.70 & 0.38 \\
\hline 303 & 12.89 & 0.30 & 0.26 & 347 & 15.03 & 0.75 & 0.35 & 391 & 14.94 & 0.82 & 0.32 \\
\hline 304 & 14.94 & 0.52 & 0.54 & 348 & 14.54 & 0.80 & 0.18 & 392 & 14.79 & 0.60 & 0.54 \\
\hline 305 & 14.95 & 0.73 & 0.25 & 349 & 14.80 & 0.58 & 0.43 & 393 & 14.75 & 1.53 & \\
\hline 306 & 14.50 & 0.49 & 0.49 & 350 & 11.47 & 0.67 & 0.14 & 394 & 13.95 & 0.62 & 0.07 \\
\hline 307 & 14.63 & 0.68 & 0.19 & 351 & 14.29 & 0.78 & 0.22 & 395 & 13.34 & 0.68 & 0.46 \\
\hline 308 & 11.85 & 0.15 & -0.07 & 352 & 14.19 & 1.05 & 0.62 & 396 & 14.60 & 0.69 & 0.20 \\
\hline 309 & 15.02 & 0.93 & 0.63 & 353 & 14.85 & 0.52 & 0.48 & 397 & 14.62 & 0.57 & 0.66 \\
\hline 310 & 14.48 & 1.61 & & 354 & 14.76 & 0.82 & 0.36 & 398 & 14.40 & 0.77 & 0.34 \\
\hline 311 & 13.82 & 0.82 & 0.32 & 355 & 14.01 & 0.45 & 0.15 & 399 & 14.57 & 0.54 & 0.48 \\
\hline 312 & 14.91 & 0.88 & 0.32 & 356 & 11.97 & 1.46 & 0.87 & 400 & 11. & 0.58 & 0.04 \\
\hline 313 & 14.79 & 1.29 & 0.97 & 357 & 14.90 & 0.86 & 0.45 & 401 & 13 & 0.34 & 0.32 \\
\hline 314 & 14.02 & 0.50 & 0.37 & 358 & 13.35 & 0.92 & 0.24 & 402 & 14.23 & 0.82 & 0.20 \\
\hline 315 & 13.12 & 0.60 & 0.35 & 359 & 13.58 & 2.34 & & 403 & 14.29 & 0.71 & 0.27 \\
\hline 316 & 13.45 & 1.13 & 0.68 & 360 & 13.56 & 0.66 & 0.45 & 404 & 13.23 & 2.16 & 1.58 \\
\hline 317 & 14.76 & 0.39 & 0.33 & 361 & 13.53 & 0.96 & 0.32 & 405 & 13.94 & 0.38 & 0.25 \\
\hline 318 & 14.74 & 0.73 & 0.22 & 362 & 14.61 & 0.71 & 0.45 & 406 & 14.75 & 0.91 & 0.44 \\
\hline 319 & 13.44 & 0.35 & -0.12 & 363 & 14.12 & 0.72 & 0.36 & 407 & 13.81 & 0.40 & 0.13 \\
\hline 320 & 14.13 & 1.31 & 0.92 & 364 & 10.68 & 0.04 & 0.08 & 408 & 13.68 & 0.74 & 0.10 \\
\hline 321 & 14.96 & 1.49 & & 365 & 13.58 & 0.94 & 0.18 & 409 & 13.58 & 0.68 & 0.56 \\
\hline 322 & 12.64 & 0.52 & 0.33 & 366 & 13.25 & 0.53 & 0.39 & 410 & 14.64 & 0.76 & 0.74 \\
\hline 323 & 15.02 & 1.16 & 0.56 & 367 & 14.86 & 0.51 & 0.35 & 411 & 14.14 & 1.50 & 1.31 \\
\hline 324 & 14.95 & 0.42 & -0.04 & 368 & 12.03 & 0.42 & 0.41 & 412 & 14.64 & 1.79 & \\
\hline 325 & 12.74 & 0.53 & 0.48 & 369 & 14.40 & 0.63 & 0.38 & 413 & 14.94 & 0.85 & 0.59 \\
\hline 326 & 13.93 & 0.50 & 0.35 & 370 & 10.35 & 0.17 & -0.02 & 414 & 14.53 & 1.53 & 1.23 \\
\hline 327 & 13.17 & 0.95 & 0.45 & 371 & 14.00 & 0.66 & 0.48 & 415 & 14.89 & 1.44 & $\ldots$ \\
\hline 328 & 13.77 & 0.33 & 0.29 & 372 & 14.97 & 0.62 & 0.52 & 416 & 14.51 & 0.54 & 0.53 \\
\hline 329 & 14.90 & 0.87 & 0.27 & 373 & 11.73 & 1.01 & 0.51 & 417 & 14.95 & 1.42 & \\
\hline 330 & 14.59 & 0.67 & -0.12 & * 374 & 14.90 & 0.44 & 0.48 & * 418 & 14.95 & 0.39 & 0.39 \\
\hline 331 & 12.05 & 0.17 & -0.26 & 375 & 11.87 & 1.18 & 1.01 & 419 & 13.51 & 1.17 & 0.89 \\
\hline 332 & 13.95 & 0.81 & 0.17 & 376 & 13.94 & 1.28 & 0.66 & 420 & 12.49 & 0.48 & 0.30 \\
\hline 333 & 14.40 & 1.93 & $\ldots$ & 377 & 13.62 & 1.57 & 1.23 & 421 & 13.27 & 0.36 & -0.42 \\
\hline 334 & 14.42 & 0.86 & 0.18 & 378 & 14.10 & 1.72 & 1.26 & * 422 & 14.26 & 0.35 & 0.08 \\
\hline 335 & 14.30 & 0.65 & 0.29 & 379 & 12.95 & 0.95 & 0.38 & 423 & 13.92 & 1.23 & 1.11 \\
\hline 336 & 14.78 & 0.81 & 0.14 & 380 & 14.02 & 0.91 & 0.30 & 424 & 13.31 & 0.55 & 0.30 \\
\hline * 337 & 13.79 & 0.70 & -0.02 & 381 & 14.09 & 0.58 & 0.36 & 425 & 14.48 & 1.30 & 0.82 \\
\hline * 338 & 12.51 & 0.69 & -0.02 & 382 & 14.73 & 0.62 & 0.48 & 426 & 14.23 & 0.41 & 0.41 \\
\hline 339 & 13.60 & 1.74 & 1.62 & 383 & 13.40 & 1.58 & 1.18 & 427 & 14.69 & 0.56 & 0.39 \\
\hline 340 & 14.91 & 0.37 & -0.08 & 384 & 14.88 & 0.58 & 0.05 & 428 & 14.21 & 1.67 & 1.22 \\
\hline 341 & 14.86 & 0.85 & 0.20 & 385 & 13.55 & 0.76 & 0.57 & 429 & 14.86 & 0.58 & 0.13 \\
\hline 342 & 13.08 & 0.66 & 0.24 & 386 & 14.63 & 1.60 & & 430 & 13.98 & 1.40 & 0.96 \\
\hline 343 & 13.24 & 0.66 & 0.02 & 387 & 13.93 & 0.82 & 0.31 & 431 & 13.37 & 0.58 & 0.34 \\
\hline 344 & 14.65 & 0.78 & 0.18 & 388 & 11.34 & 0.72 & 0.51 & * 432 & 14.44 & 0.39 & 0.07 \\
\hline
\end{tabular}

$E_{B-\nu}$, as expected for SW Vel (Turner 1979). However, there is some evidence in Figure 15 for a spread in reddening values, so the data were analyzed using the variable-extinction method. The first step involved the derivation of a reddening line for the field of slope $E_{U-B} / E_{B-V}=0.84 \pm 0.01$ from an analysis of $(i)$ the upper envelope of 13 reddened early B-type stars ( slope $0.84 \pm 0.04$ ), (ii) a sample of 62 stars ( slope $0.83 \pm$ 0.01 ) apparently reddened from the A2 kink in the intrinsic color-color relation for dwarfs, and (iii) the color excesses (slope $0.85 \pm 0.03$ ) derived for spectroscopically-observed stars in the field (Turner 1979). Such a large reddening slope for this field is rather unusual, implying by analogy with the results of Turner (1989) that the differential reddening is produced locally by dust clouds associated with Gould's Belt. The variable-extinction analysis confirms this suspicion. None of the unreddened or little-reddened stars in the field have distance moduli in excess of $V_{0}-M_{V} \approx 8.9$, which corresponds to a distance of $\sim 600 \mathrm{pc}$ for the dust clouds.
A variable-extinction diagram for reddened B- and A-type stars in the SW Vel field is presented in Figure 16. We have included data for OB stars in the larger environs of the $\mathrm{Ce}$ pheid, with the $U B V$ data for these stars taken from Klare \& Neckel ( 1977) and Schild, Garrison \& Hiltner (1983). Absolute magnitudes $M_{V}$ for these stars were derived as in Turner (1986) by using Klare \& Neckel's $\mathrm{H} \beta$ indices with the $W(\mathrm{H} \gamma)$ calibration of Millward \& Walker (1985), and by adjusting the final values for rotational effects assuming $V \sin i$ values for each star typical of the average for the star's spectral type ( $\mathrm{Fu}-$ kuda 1982) or, if the star was designated spectroscopically as $\mathrm{n}$ or nn, assuming that $V \sin i>250 \mathrm{~km} \mathrm{~s}^{-1}$. This technique has been applied to B stars in NGC 129 by Turner, Forbes, \& Pedreros (1992) with reasonable success. For the SW Vel sample it has the effect of modifying the membership selection for stars considered to belong to the two associations identified by Turner (1979). Stars KS 204, 224, and 244 of the 26 star sample considered by Turner (1979, Table 1) to belong to Vel 


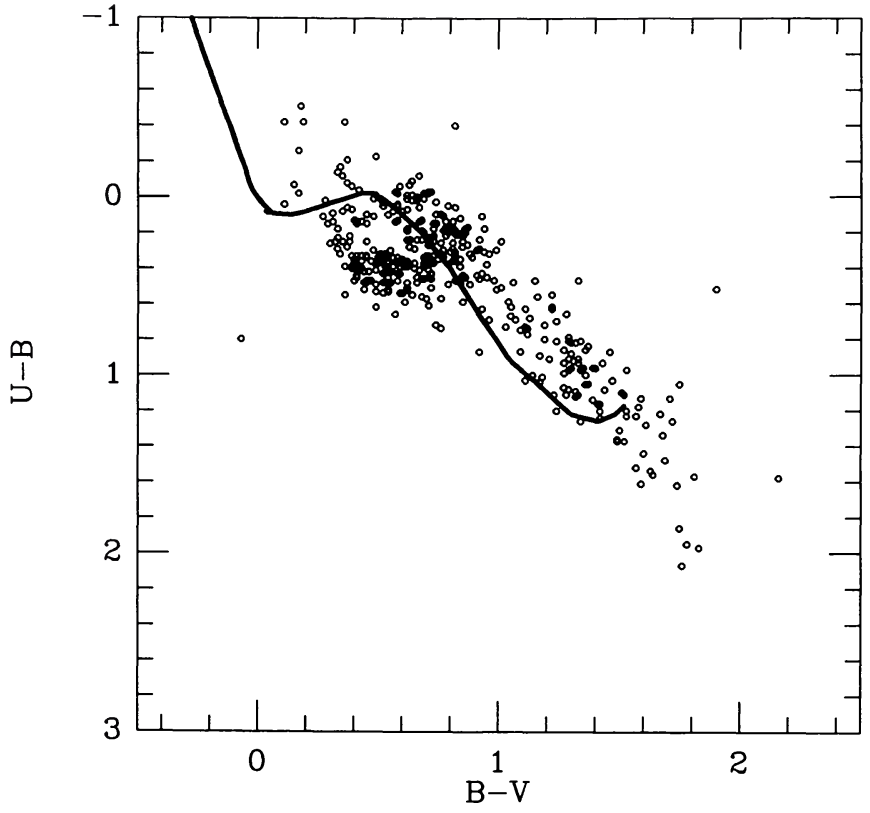

FIG. 15.-Color-color plot for stars in the SW Vel field. The solid line is the intrinsic relation for dwarfs.

OB1 appear to be part of the more distant association believed to contain SW Vel, while stars KS 203, 227, and 256 of the latter 16 star sample (Turner 1979, Table 2) are clearly identified as background objects. The remaining sample of 16 stars believed to be associated with SW Vel actually becomes more homogeneous with these changes, consisting now entirely of $B$ stars with no O-type members. As an extension of the Vela OB association nomenclature used in the survey by Slawson \& Reed (1988), we designate this group as Vel OB5.

The interpretation of Figure 16 is fairly straightforward. All stars in the field more distant than $\sim 600 \mathrm{pc}$ are reddened by $E_{B-V}(\mathrm{~B} 0)=0.30$, and reddenings in excess of $E_{B-V}(\mathrm{~B} 0)=$ 0.50 appear only beyond $V_{0}-M_{V} \approx 12.0(\sim 2.5 \mathrm{kpc})$. There is a distinct lower envelope (or upper limit in distance modulus) for ZAMS-fitted stars in the reddening interval $E_{B-V}=$ $0.3-0.5$, and the distance moduli of these stars (designated by asterisks in Table 8) closely match those derived for luminous members of Vel OB5. This result provides additional evidence for the distinct nature of Vel OB5 as a physical group and increases the likelihood of SW Vel being an association member.

Various regression and non-parametric straight line fits were made to the ZAMS-fitted and $\mathrm{H} \beta$-derived data in Figure 16 for stars identified here with Vel OB5. These include the stars denoted by asterisks in Table 8 and all but KS 206 (a Be star) of the luminous stars described above. The derived values of $R$ were $3.38 \pm 0.40$ for the 15 spectroscopically observed stars, $3.14 \pm 0.14$ for the 22 ZAMS-fitted stars, and $3.23 \pm 0.12$ for the combined sample. An adopted best value of $R=3.20 \pm$ 0.09 was assumed to apply to the dust extinction in this field. The corresponding distance modulus for Vel OB5 is $V_{0}-$
$M_{V}=11.99 \pm 0.15$ s.d. $( \pm 0.03$ s.e. $)$, which corresponds to a distance of $2.50 \mathrm{kpc}$.

The average space reddening for the five stars lying within $3^{\prime}$ of SW Vel which have well-established color excesses in the $E_{B-V}=0.3-0.5$ interval is $\left\langle E_{B-V}\right\rangle=0.35 \pm 0.03$ s.d. Within a larger 5 ' radius interval there are 12 stars of comparable reddening with $\left\langle E_{B-V}\right\rangle=0.38 \pm 0.02$ s.d. We have adopted the latter value for the field reddening of SW Vel. The corresponding reddening appropriate for the Cepheid is $E_{B-V}($ Cepheid $)=$ $0.35 \pm 0.02$.

A reddening-corrected color-magnitude diagram for likely members of Vel OB5 is given in Figure 17. The parameters for SW Vel as a member of this group are $\left\langle M_{V}\right\rangle=-5.09 \pm 0.07$ and $(\langle B\rangle-\langle V\rangle)_{0}=+0.80 \pm 0.02$. The period-luminosity and period-color relations of van den Bergh (1977) predict corresponding values of $\left\langle M_{V}\right\rangle=-5.15 \pm 0.26$ and $(\langle B\rangle-\langle V\rangle)_{0}=$ $+0.93 \pm 0.06$ for a Cepheid of identical period. The agreement in luminosity estimates is excellent, although there is clearly some disagreement in the derived intrinsic colors. Given the similarity in derived colors for WZ Sgr and SW Vel, which have similar pulsational periods, this discrepancy is probably not a serious concern. In any case, there is no possibility of deriving a much smaller space reddening for SW Vel with the photometric data for surrounding B stars.

The luminous members of Vel OB5 identified here are giants and supergiants which range in spectral type from $\mathrm{B} 0$ to B2. These stars generally lie several degrees distant from SW Vel. The immediate vicinity of SW Vel contains no stars bluer than $(B-V)_{0}=-0.23$, which corresponds to an earliest main-

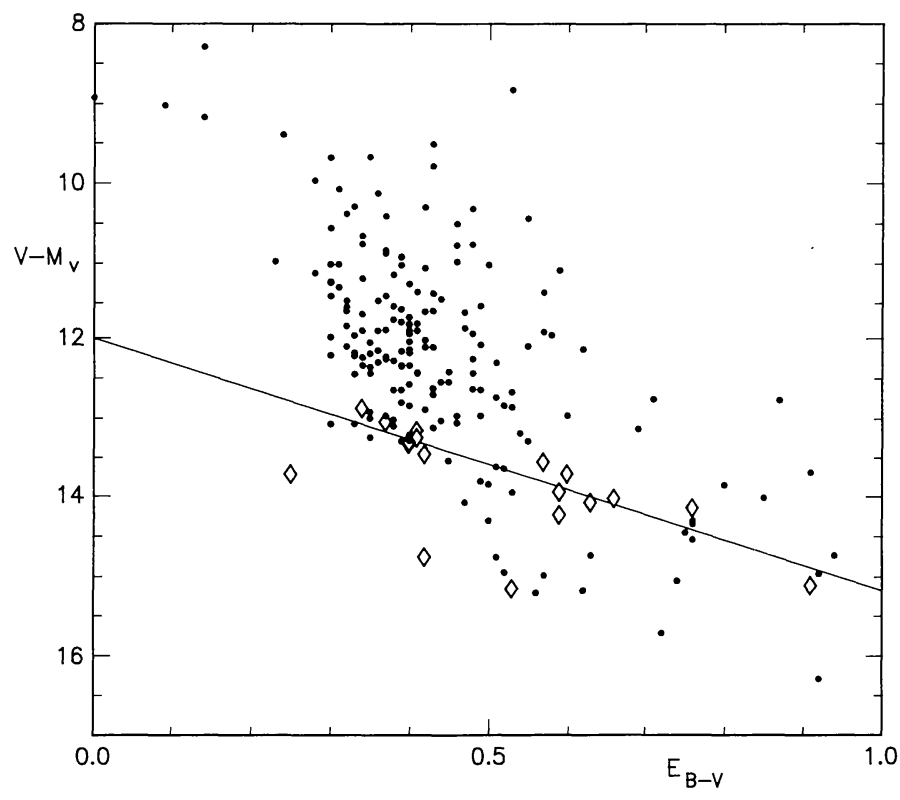

FIG. 16.- Variable-extinction diagram for stars in the SW Vel field (circles) and for luminous stars in the larger environs of the Cepheid (diamonds). The line of slope $R=3.2$ represents a best fit to stars identified as possible members of Vel OB5 (see text). 


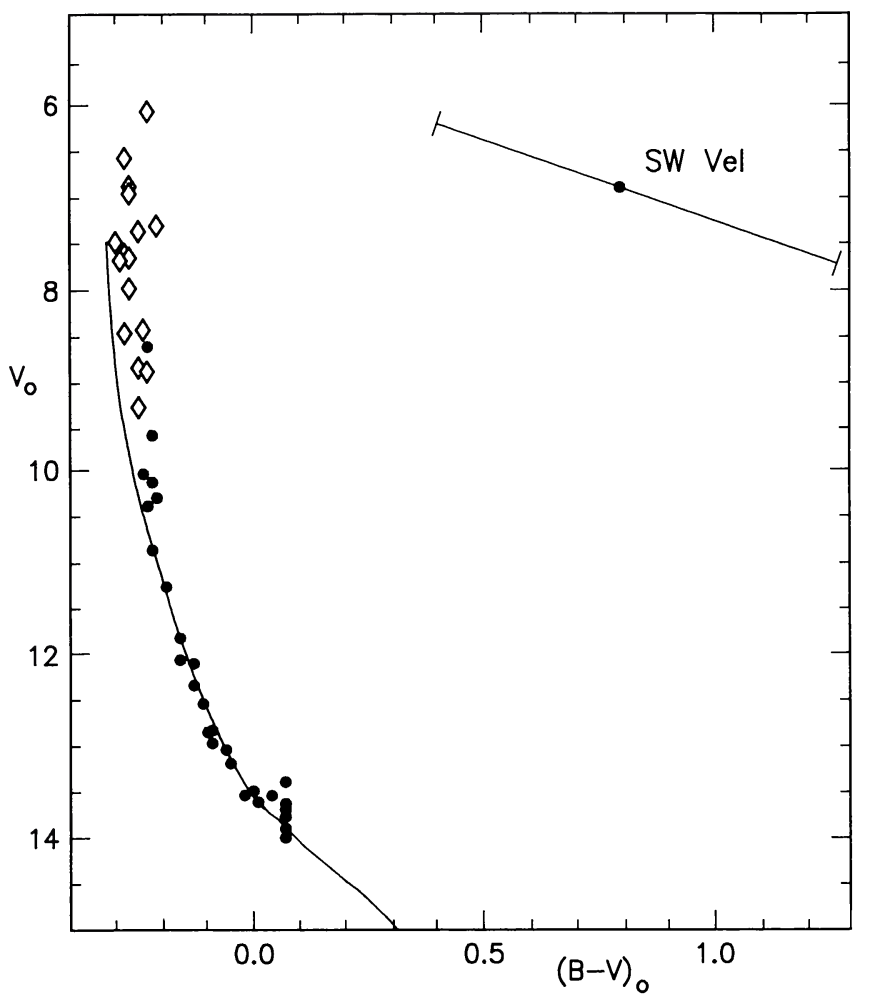

FIG. 17.-A reddening-free color-magnitude diagram for stars identified as possible members of Vel OB5, with symbols as in Fig. 16. The ZAMS is plotted for $V_{0}-M_{V}=11.99$.

sequence spectral type of B2 for the Vel OB5 members nearest the Cepheid. This result implies the existence of different age subgroups in Vel OB5, with the stars surrounding SW Vel having ages of about $2.1( \pm 0.3) \times 10^{7} \mathrm{yr}$ according to the cluster age calibration of Mermilliod (1981). This makes them roughly coeval with SW Vel, which is further support for the idea that SW Vel is a member of the Vel OB5 subgroup identified in its surrounding. Additional photometry as well as radial velocity data are clearly essential for confirming this possibility. Photometrically identified members of Vel OB5 in the SW
Vel field exhibit no particular spatial clustering, although several are located in a sparse grouping a few minutes of arc south of the Cepheid. It is certainly conceivable that the remains of the parent cluster for SW Vel no longer exist as a recognizable entity, although the larger region beyond our survey field does contain several unstudied open clusters.

\section{CONCLUSIONS}

The results of the present study are summarized in Table 9 , which contains successful photometric detections of associated B stars for three of the four program Cepheids. In the case of WZ Sgr, the Cepheid is found to lie in a previously undetected open cluster, which is strongly indicated to be physically associated with the Cepheid. In the case of GT Car, possible companions to the Cepheid are detected even though they are at the limit of the present survey. The final case, that for SW Vel, confirms expectations from a previous study (Turner 1979) as well as many of the conclusions found in Paper III. Most longperiod Cepheids are not situated in the cores of rich associations, and in the older association subgroups where Cepheids are expected the surface density of B stars may be too low for significant numbers of such objects to be situated within close proximity of a Cepheid.

The present study also indicates how previous photometric searches might have been modified so that there was a greater chance of success. As a first step, the calibration of the photographic photometry can be improved significantly with more extensive and better quality standard sequences. This helps to reduce the random and systematic errors in the data to the point where variable-extinction analyses become feasible. Our survey fields are large enough that variable interstellar extinction can have significant effects on the observed stellar magnitudes and colors. All of the successful detections presented in this paper resulted from a proper consideration of these effects, which indicates how important this step can be.

Further improvements might result from CCD imaging, rather than photographic imaging, of the Cepheid fields. However, the surface density of associated B stars to long-period Cepheids in the present study is clearly low enough to make large field imaging essential for success. Photographic surveys are still useful in this regard and have the added advantage of being less susceptible to potential systematic errors in color, an

TABLE 9

SUMMARY OF RESULTS

\begin{tabular}{|c|c|c|c|c|c|c|c|c|}
\hline \multirow[b]{2}{*}{ CEPHEID } & \multirow[b]{2}{*}{$P$ (days) } & \multicolumn{4}{|c|}{ ASSOCIATED GROUP } & \multicolumn{3}{|c|}{ CEPHEID PARAMETERS } \\
\hline & & Name & $V_{0}-M_{V}$ & $d(\mathrm{kpc})$ & Turnoff & $E_{B-V}$ & $(\langle B\rangle-\langle V\rangle)_{0}$ & $\left\langle M_{V}\right\rangle$ \\
\hline XY Car & 12.437 & & & & & & & \\
\hline GT Car & 13.162 & Car Anon OB & $15.04^{\mathrm{a}}$ & 10.17 & B3 & 0.66 & +0.56 & $-4.43^{b}$ \\
\hline WZ Sgr ...... & 21.850 & C1814-191a & 11.26 & 1.79 & B2.5 & 0.56 & +0.84 & -5.07 \\
\hline SW Vel ...... & 23.442 & Vel OB5 & 11.99 & 2.50 & $\mathrm{~B} 2$ & 0.35 & +0.90 & -5.09 \\
\hline
\end{tabular}

\footnotetext{
${ }^{a}$ Based upon adopted luminosity.
}

${ }^{b}$ Adopted value. 
annoying problem for some types of CCD observing (see Turner 1990). Progress in CCD imaging should ultimately remove both disadvantages for surveys of the present type.

We wish to thank the directors of the Cerro Tololo and Las Campanas observatories for their help and hospitality, and Bob Garrison for his generous allotments of observing time with the University of Toronto's $0.6 \mathrm{~m}$ Chile telescope. We are indebted to Elmar Brosterhus for obtaining the 1978 and 1979 plates, and to David Duncan for assistance with the finder charts and figures. This investigation was supported in part by funds awarded (to D.G.T. and D.F.) through the Natural Sciences and Engineering Research Council of Canada (NSERC), and was initiated during the tenure of a NSERC University Research Fellowship by D.G.T.
Burki, G. 1978, A\&A, 62, 159

Caldwell, J. A. R., \& Coulson, I. M. 1987, AJ, 93, 1090

Eggen, O. J. 1982, ApJS, 50, 199

Fernandez, J. A., \& Salgado, C. W. 1980, A\&AS, 39, 11

Fernie, J. D. 1962, ApJ, 135, 298 1963, AJ, 68, 780

Fukuda, I. 1982, PASP, 94, 271

Gieren, W. P. 1988, PASP, 100, 262

Harris, G. L. H. 1976, ApJS, 30, 451 1980, in IAU Symp. 85, Star Clusters, ed. J. E. Hesser (Dordrecht: Reidel), 195

Hartwick, F. D. A., \& Hesser, J. E. 1973, ApJ, 183, 883

Hiltner, W. A. 1956, ApJS, 2, 389

Houk, N., \& Cowley, A. P. 1975, University of Michigan Catalogue of Two-Dimensional Spectral Types for the HD Stars, 1 (Ann Arbor: Univ. Michigan Press)

Humphreys, R. M. 1978, ApJS, 38, 309

Kippenhahn, R., \& Smith, L. 1969, A\&A, 1, 142

Klare, G., \& Neckel, T. 1977, A\&AS, 27, 215

Madore, B. F. 1975, ApJS, 29, 219

Maeder, A., \& Meynet, G. 1988, A\&AS, 76, 411

Mermilliod, J. C. 1981, A\&A, 97, 235

Millward, C. G., \& Walker, G. A. 1985, ApJS, 57, 63

Neckel, T., \& Klare, G. 1980, A\&AS, 42, 251

Parsons, S. B., \& Bell, R. A. 1975, in Multicolor Photometry and the Theoretical HR Diagram, ed. A. G. D. Philip \& D. S. Hayes (Schnectady: Dudley Obs. Press), 73

Parsons, S. B., \& Bouw, G. D. 1971, MNRAS, 152, 133

Racine, R. 1968, AJ, 73, 233

\section{REFERENCES}

Reed, B. C., Turner, D. G., \& Scrimger, J. N. 1986, JRASC, 80, 203

Schild, R. E., Garrison, R. F., \& Hiltner, W. A. 1983, ApJS, 51, 321

Sher, D. 1964, MNRAS, 129, 237

Slawson, R. W., \& Reed, B. C. 1988, AJ, 96, 988

Stephenson, C. B., \& Sanduleak, N. 1971, Publ. Warner \& Swasey Obs., Vol. 1, No. 1

Turner, D. G. 1979, A\&A, 76, 350

- 1984, PASP, 96, 422

- 1986, AJ, 92, 111

- 1989, AJ, 98, 2300

- 1990, PASP, 102, 1331

Turner, D. G., Forbes, D., \& Pedreros, M. 1992, AJ, 104, 1132

Turner, D. G., Leonard, P. J. T., \& English, D. A. 1987, AJ, 93, 368

Turner, D. G., \& Welch, G. A. 1989, PASP, 101, 1038

Tzarevsky, G. S. 1970, Nauch. Inform. Akad. Nauk. S.S.S.R., 16, 117

Ulrich, R. 1971, ApJ, 168, 57

van den Bergh, S. 1977, in IAU Colloq. 37, Décalages vers le Rouge et Expansion de l'Univers, ed. C. Balkowski \& B. E. Westerlund (Paris: CNRS), 13

van den Bergh, S., Bosterhus, E. B. F., \& Alcaino, G. 1982, ApJS, 50, 529 (Paper I)

van den Bergh, S., Younger, P. F., Brosterhus, E. B. F., \& Alcaino, G. 1983, ApJS, 53, 765 (Paper II)

van den Bergh, S., Younger, P. F., \& Turner, D. G. 1985, ApJS, 57, 743 (Paper III)

Walborn, N. R. 1973, AJ, 78, 1067

Weaver, H. 1962, in Handbuch der Physik, 54, Astrophysics V: Miscellaneous, ed. S. Flügge (Berlin: Springer-Verlag), 130 